\title{
A Study of the Noise Mechanisms of Transonic Blade-Vortex Interactions
}

\author{
Lyrintzis, A. S.* and Xue, Y.**
}

Aerospace Engineering \& Mechanics

University of Minnesota, Minneapolis, MN 55455

\section{Abstract \\ Transonic Blade-Vortex Intsractions (BVI) are simulated numerically and the noise mechanisms.} are investigated. The two-dimensional high frequency transonic small disturbance equation is solved numerically (VTRAN2 code). An ADI scheme with monotone switches is used; viscous effects are included on the boundary and the vortex is simulated by the cloud-in-cell method. The Kirchhoff method is used for the extension of the numerical two-dimensional near-field aerodynamic results to the: linear acoustic three-dimensional far-field. The viscous effect (shock/boundary layer interaction) on BVI is investigated. The different types of shock motion are identified and compared. Two important disturbances with different directivity exist in the pressure signal and are believed to be related to the fluctuating lift and drag forces. Noise directivity for different cases is shown. The maximum radiation occurs at an angle between $60^{\circ}$ and $90^{\circ}$ below the horizontal for an airfoil-fixed coordinate system and depends on the details of the airfoil shape. Different airfoil shapes are studied and classified according to the BVI noise produced.

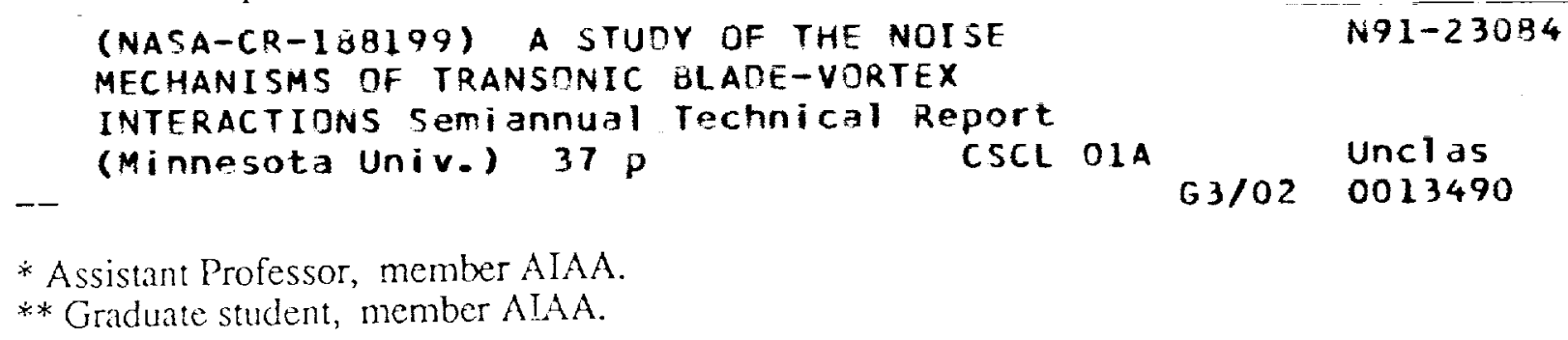




\section{Introduction}

Among the several types of helicopter noise [1], that due to Blade Vortex-Interactions (BVI) is one of the most important. BVI is the aerodynamic interaction of a rotor blade with the trailing vortex system generated by preceding blades as shown in Fig. 1 (from reference [2]). It usually occurs during helicopter descent, or low speed maneuvers. It is loud, impulsive in character, and tends to dominate the other sources when it occurs, as shown experimentally (e. g. Schmitz and Yu [3]). Also, very complicated BVI patterns arise from tilt-rotor aircraft [4]. Interactions generate the most significant noise when they are intrinsically unsteady, as when the vortex is exactly parallel to the blade, or when the vortex is nearly parallel to the blade (a vertical interaction is steady for 2-D blade). Incompressible BVIs have been successfully treated in the past (e. g. reference [5]). For typical helicopter cases though, it was shown in references [6] anc [7] that the aerodynamics and aeroacoustics of the interactions are intrinsically transonic. In such cases the flow can be initially modeled by two-dimensional unsteady transonic flow (fig. 2).

Unsteady transonic flow problems have been solved numerically in the past. The low frequency approximation of the unsteady two-dimensional Transonic Small Disturbance (TSD) equation was first solved by Ballhaus and Goorjian [8] and the LTRAN2 code was created. Since then, the code has been updated to include high frequency effects [9], viscosity [10], monotone switches [11] and second order effects [12]. However, the acoustic waves resulting from the unsteady motion have not been adequately studied.

Two-dimensional transonic BVI was first studied computationally in the near-and mid-field by George and Chang $[6,7]$ who used the high frequency transonic small-disturbance equation, including regions of convected vorticity. References $[6,7]$ also contain detailed discussions of the background and formulation of the transonic BVI problem. A comprehensive code, VTRAN2 was developed [13, 14] as a modification of LTRAN2 to include the vortex. The vorticity is bilinearly distributed inside a vortex core and branch cuts are introduced in the $\mathrm{x}$-direction. The vortex can either follow a prescribed path, or can be convected with the free stream. A new look at the physics of the acoustics of unsteady transonic flow was ;iven in reference [15]. In references [16-24] th: two-dimensional transonic BVI problem is also solved using the small disturbance theory and the more complex Euler and thin-layer 
Navier Stokes equations. Also Baeder et al. [22, 23] and Liu et al. [24] presented some near and mid field results. At great distances from the airfoil though, the waves become very difficult to follow because of numerical diffusion and dispersion errors.

Kirchhoff's method was introduced [14, 25-29] to extend the numerically calculated nonlinear aerodynamic results to the linear acoustic far-field. This method uses a Green's function for the linearized governing equation to derive a representation for the solution in terms of its values and derivatives on a closed surface $S$ in space, which is assumed to include all the nonlinear flow effects and noise sources. The potential and its derivatives can be numerically calculated from a nonlinear aerodynamic code (e. g. VTRAN2). The Kirchhoff method has the advantage of including the full diffraction effects and eliminates the erroneous propagation of the reactive near-field.

In this paper we examine the noise due to BVI. An existing code (VTRAN2) was enhanced to include monotone switches and viscous effects. The viscous effects (shock/boundary layer interaction) on BVI noise are studied. The resulting noise because of the different types of shock-wave motion types in the near- and the far-field is investigated and the different resulting disturbances are analyzed. The noise mechanisms are explained physically and the relation between the noise signal and oscillating lift and drag forces is shown. The complicated directivity patterns of BVI noise are also studied. Different airfoil shapes are studied and classified according to the produced BVI noise.

\section{The Numerical Method (VTRAN2)}

VTRAN2 is a code $[13,14]$ developed for analyzing the interactions of convected regions of vorticity with aiffoils using transonic small disturbance theory. It is based on the ADI implicit scheme of the LTRAN2 code [8] with the inclusion of the high frequency term as described in reference [9] and the addition of regions of convected vorticity using the cloud-in-cell and multiple branch-cut approach. The code was modified to include viscosity [8] and monotone switches [10].

The governing equation for the unsteady transonic small-disturbance potential and the boundary conditions can be found in various references (e. g. $[30,25]$ ). The classical Kutta condition is satisfied by this small-disturbance formulation. We are interested in cases for which the reduced frequency range is less than 4, which is the limit for the application of the Kutta condition [31]. A finite vortex core is used (clrud-in-cell method) for reasons of computational stability. The core has a finite square shape 
limited by grid-lines and the voricity is bilinearly distributed inside. Thus, several branch cuts (in the $x$ direction) are introduced. The vortex can have a free path (convected by the flow) or a prescribed path (miss distance $\mathrm{y}_{\mathrm{V}}=$ constant, vortex velocity $=\mathrm{U}_{\mathrm{O}}$ ). Details of the theoretical formulation were given by Chang [32] and Lyrintzis [14].

For the viscosity calculations the viscous ramp method (wedge) is used. The viscous ramp model simulates the shock/boundary layer interaction by placing a wedge-nosed ramp at the base of the shock to obtain the reduced shock pressure rise. The surface geometry must be augmented by the ramp model by adding an extra viscous term in the boundary condition. Details of the calculation of that viscous term can be found in reference [33]. The ramp model was derived for steady-state computations. However, it can be incorporated into unsteady computations in a quasi-steady fashion. Thus, the model is valid for low frequencies, and its use in high frequency problems such as BVI can only give some qualitative information about the effect of viscosity with almost no additional CPU time. The more complicated and CPU time-consuming lag-entrainment method which was also incorporated in our code was not used, because it was not superior to the wedge model for unsteady cases [33].

An alternating direction im $_{r}$ licit (ADD) method is used for the solution of the equation, where the high frequency term is added in the y-sweep. An approximate factorization technique with monotone switches [11] is used for the steady calculation, which provides a start-up solution. Special care is taken for the conservative differentiation along the uneven mesh.

A $213 \times 199$ mesh is used for the calculations. The computational mesh points are clustered more densely near and in front of the airfoil and are stretched exponentially from the near airfoil region to about 200 chords from the airfoil in the $\mathrm{x}$ - and 400 in the $y$-direction. More mesh points are added in the $y$-direction for the more accurate evaluation of the normal derivatives on the Kirchhoff surface. The VTRAN2 code was shown to agree well with other, more complex approaches including Euler and thinlayer Navier-Stokes computations [7]. The code has a high vectorization level and the CPU time for each two-dimensional case on a Cray- 2 computer is about 4 minutes for 800 time-marching steps.

\section{Kirchhoff's Method for the Far-Field}

In the past acoustic analogy has been used for the evaluation of noise signals. This approach starts from the calculation of the nonlinear near- and mid-field and the far-field is found from vitice and 
volume integrals of near- and mid-field flow and body surfaces. We should note that there are substantial difficulties in including the nonlinear quadrupole term (which requires second derivatives) in the volume integrals, especially around shock surfaces. Thus, a lot of investigators use near-field data only on the blade surface, which is less accurate as shock surfaces are not included in the calculation.

Kirchhoffs method includes the calculation of the nonlinear near-and mid-field with the far-field solutions found from a linear Kirchhoff formulation evaluated on a surface surrounding the nonlinearfield. This method provides an adequate matching between the aerodynamic nonlinear near-field and the acoustic linear far-field. The full nonlinear equations are solved in the first region (near-field), usually numerically, and a surface integral of the solution over the control surface gives enough information for the analytical calculation in the second region (far-field). The advantage of the method is that non linear effects (e. g. shock waves) are accounted for. Also, the surface integrals and the first derivatives needed can be easily evaluated from the near-field CFD data; full diffraction and focusing effects are included while eliminating the propagation of the reactive near-field.

The Kirchhoff equation for a moving surface was originally derived by Morgans [34]. A Green's function approach will be used to rederive the Kirchhoff formula in a coordinate system fixed to the airfoil which moves with velocity $\mathrm{U}_{\mathrm{O}}$. The Green's function approach was introduced by Morino [35, 36]. Farassat and Myers [37] rederived the Kirchhoff equation for an arbitrarily moving piecewise smooth deformable surface using generalized derivatives. A very brief discussion of the Kirchhoff formulation is given in the following paragraphs; for more details the reader is referred to the above references, and also references $[14,26]$.

A Green's function for the linearized governing equation is used to derive a representation for the solution in terms of its values and derivatives on a closed surface $S$ in space, which is assumed to include all the nonlinear flow effects and noise sources. A full three-dimensional formulation is used, because the Green's function is simpler in this case, and because the method can be easily extended to include spanwise variations to model three-dimensional BVI. The pressure distribution at a point $\left(x_{0}, y_{0}, z_{0}\right)$ outside a rigid fixed surface is

$p\left(x_{0}, y_{0} \cdot z_{0}, t\right)=-\frac{1}{4 \pi} \int_{S}\left[\frac{1}{r_{0}} \frac{\partial p}{\partial n_{0}}+\frac{1}{c_{0} r_{0} \beta^{2}} \frac{\partial p}{\partial t}\left(\frac{\partial r_{0}}{\partial n_{0}}-M \frac{\partial x_{o}^{\prime}}{\partial n_{O}}\right)+\frac{p}{r_{0}^{2}} \frac{\partial r_{0}}{\partial n_{0}}\right]_{\tau}\left(S_{O}^{\prime}\right.$ 
where

$$
\begin{gathered}
r_{0}=\left\{\left(x-x^{\prime}\right)^{2}+\beta^{2}\left[\left(y-y^{\prime}\right)^{2}+\left(z-z^{\prime}\right)^{2}\right]\right\}^{1 / 2} \\
\tau=\frac{\left[r_{O}-M\left(x-x^{\prime}\right)\right]}{c_{O} \beta^{2}} \\
\beta=\left(1-M^{2}\right)^{1 / 2}
\end{gathered}
$$

where " " " denotes a point on the Kirchhoff surface, subscript o denotes the transformed values using the well known Prandil-Glauert transformation:

$$
x_{0}=x, y_{0}=\beta y, z_{0}=\beta z
$$

$\mathrm{n}$ is the outward vector normal to the surface $S$, and subscript $\tau$ implies the evaluation at the retarded time $t_{1}=t-\tau$.

Thus, the values of the potential and its normal derivatives on an arbitrary surface around the spanwise extent of an arbitrary flow are enough to give the far-field radiation at any arbitrary external point." In our work we use as a control volume a rectangular box (Fig. 3) coinciding with mesh points in order to simplify the computation. The potential and its derivatives can be numericaily calculated from an aerodynamic near-field code; then equation (1) is used to evaluate the solution in the far-field. Thus, the solution is integrated on a surface in the mid-field and nonlinear effects (e. g. shock waves) in the near-field are fully accounted for.

Since Kirchhoff's method assumes that linear equations hold outside this control surface $S$, the latter must be chosen large enough to include the region of nonlinear behavior. However, due to increasing mesh spacing the accuracy of the numerical solution is limited to the region immediately surrounding the moving blade. Thus $S$ cannot be be too large, because the numerical evaluation of the derivatives needed (equation 1 ) is not accurate at large distances. Therefore, a judicious choice of $S$ is required for the effectiveness of the Kirchhoff method. A rectangular box-shaped surface (Fig. 3 ) is used for the calculations. The VTRAN2 code is used to calculate the solutions on the surface S. The ylimits of $S$ for our calculations are varied over a range from $y_{S}=0.25$ to 4.00 chords distance from the airfoil. Higher Mach numbers yield higher optimum values for $y_{s}$ because of stronger nonlinearities in the larger lateral extent of the flow region. The $x$-limits for $S$ were also varied between 0.15 and 0.50 chords and, similarly, values of 0.25 chords upstream and downstream of the leading and trailing edges respectively, are chosen. 
Strip theory approximation :s used; that is, the two-dimensional VTRAN2 solution is applied on different segments of the blade in a stripwise manner. Blade segments ranging from two to sixteen in aspect ratio are used. Usually mesh limitations keep the Kirchhoff surface close enough to the blade, where the two-dimensional strip theory solution is still valid. By making calculations with or without the inclusion of the tip surfaces we found [27] that they have only a small effect; thus they were neglected for most of the calculations.

\section{Types of Unsteady Shock Motion}

Tijdeman [38] showed experimentally, using an oscillating flap, that varying airfoil surface boundary conditions can give three different types of unsteady shock motion:

Type A shock motion, where the shock at the rear of the supersonic region merely moves back and forth with concurrent changes in strength.

Type $B$ shock motion, where the shock moves similarly to type A, but disappears temporarily during the unsteady motion.

Type $\mathrm{C}$ shock motion, where the supersonic region disappears, but a shock wave leaves the airfoil and propagates forward to the far-field.

The above three types of unsteady shock motions affect heavily the characteristics (e. g. lift, drag) of all unsteady transonic flows. The type of shock motion that occurs in a given situation depends on the flow characteristics (e. g. free-stream Mach number, airfoil shape, amplitude and frequency of the unsteady motion). These types of shock motion can even be observed in steady airfoils with severe flow separation downstream of the shock waves. Their existence in BVI has been verified by different experiments and calculations (e. g. Tangler's experiments [39]).

\section{Results and Discussion}

Some mid-field calculations for BVI are performed using VTRAN2 with a refined mesh to follow the waves of interest. Then the Kirchhoff method is used to examine the noise at the far-field. The calculations are made in an airfoil-fixed reference frame. A more detailed discussion of the coordinate systems used for BVI calculations is given in reference [25].

We use a NACA $64 A 006$ airfoil, the vortex strength was $C_{l v}=0.4$ ( $C_{l v}$ is a nondimensional measure of the vortex strength: $\left.\mathrm{C}_{v}=2 \Gamma / c U_{0}\right)$ and the vortex miss-distance $y_{0}=-0.5$ chords, for a fixed 
vortex path. The initial vortex position is $x_{0}=-9.51$ chords and the free stream velocity is one (arbitrary units) so the vortex passes below the airfoil leading edge at time $\mathrm{T}=9.51$. The Mach numbers of 0.875 , 0.854 , and 0.822 comespond to shock wave motions of types $\mathrm{A}, \mathrm{B}$, and $\mathrm{C}$, respectively, as also shown in reference [7]. The three different types of the unsteady shock motion are thus studied. For the Kirchhoff surface (Fig. 3) we used a span of 8 chords, $x_{S}=0.25$ chords and $y_{S}=3.5,2.5$ and 1.9 chords for the three types A, B aid C, respectively. Note that a larger $\mathrm{y}_{\mathrm{S}}$ is required for higher Mach numbers, because the $y$-extent of the nonlinear region of the flow increases, as expected from the scaling laws of transonic flow.

Fig. 4 shows the effect of the grid on the lift coefficient $\mathrm{Cl}_{\mathrm{l}}(\mathrm{T})$. A standard mesh $213 \times 119$ is compared to a finer in the $y$-direction mesh $213 \times 199$. The results show that the fine mesh produces a smoother 'solution. Smoother solutions are also produced for the pressure coefficient at different points, especially in the far-field using the Kirchhoff method. Finer meshes were also tried, but the results were not substantially changed. Thus, the fine mesh $(213 \times 199)$ will be used in the subsequent calculations.

Figs 5,6 and 7 show the effect of viscosity in the calculations. The pressure coefficient $C_{p}(T)$ at point $P(-0.3000,-0.17478)$, the lift coefficient $C_{l}(T)$ and the drag coefficient $C_{d}(T)$ are plotted for a type A shock motion, for viscous and nonviscous calculations. We can see only a slight influence of the effect of viscosity. Since viscosity is added as an extra boundary condition to model shock/boundary layer interaction, we expect the influence to be stronger with the increase of the strength of the shock. Thus, the effect of viscosity is lower for the types B and C (not shown) and zero for subcritical cases. The following results include the effect of the viscosity.

Figs 8 and 9 show the $C_{p}(T)$ signal of the three types of unsteady shock motion in the mid-field (point P) from VTRAN2 and in the far-field (point $O, r=20$ chords, $\theta=30^{\circ}$ ), as shown in Fig. 3 , using Kirchhoff's method. The signal for the higher Mach number (type A) propagates upstream slower, so it takes longer time to arrive at the same point (Fig. 9). The signal consists of three disturbances (I, II, III) as also shown in references $[25,26]$. The primary disturbance $I$ is the main BVI noise and it originates at the airfoil when the vortex passes below the leading edge. It is believed to be related to the fluctuating lift coefficient $\mathrm{Cl}_{1}$. The secondary disturbance II corresponds to the unsteady shock motion and depends on the motion of the entire supersonic region induced by the vortex passage. It originates at the airfoil at a later time, and depends heavily on the type of shock motion. It is believed to be somehow related to 
the fluctuating drag coefficient $C_{d}$. The time delay of disturbance II decreases with decreasing Mach number and disappears in subcritical cases. The directivity of the two disturbances is very different as will be shown later. The existence of the second disturbance was observed computationally by George and Chang [7] and was also verified experimentally by Caradonna et al. [40] and Shenoy [41], and computationally by George and Lyrintzis [25, 26], Owen and Shenoy [20] and Liu et al. [24]. For example, in reference 41 Schlieren experiments were performed for a rotor and a second disturbance was seen to propagate in the far-field as postulated by computational results. Disturbance III is considered to be a standing disturbance due to the vortex passage and it is not a propagating wave. Thus this disturbance disappears as we move from the mid-field (Fig.8) to the far-field (Fig. 9).

From Figs 8 and 9 we can see that disturbance I increases slightly with increasing Mach number. We should also bear in mind that the definition of $C_{p}$ includes division by $M^{2}$, so the effect of the Mach number is stronger than it appears in the above figures. Disturbance $\Pi$ exists also for type $A$ and $B$ shock motions, because it is caused by the movement of the entire supersonic pocket. and seems to be magnified as we move into the far-field. It also appears to be decreasing as we move from type $\mathrm{C}$ to type A. However, if we measure it from peak to peak (instead of just reading the max value) it still increases, but at a lower rate thar disturbance I. Disturbance III is almost the same for the three cases, which seems reasonable since the same vortex strength is used.

Fig. 10 shows the lift coefficient $C_{I}(T)$ for types $A, B$ and $C$. We can see that their shapes relate well to the first disturbances shown in previous figures. Specifically the total $\mathrm{Cl}_{\mathrm{l}}$ change for the type $\mathrm{A}$ shock motion is much higher than that for type $\mathrm{C}$. Thus, we can deduce that disturbance $\mathrm{I}$ is most probably related to $\mathrm{Cl}_{1}$.

The drag coefficient $C_{d}$ will be discussed next. It is well known that in uniform subcritical inviscid flow $\mathrm{C}_{\mathrm{d}}$ is zero. It should be noted though, that $\mathrm{C}_{\mathrm{d}}$ is not zero for subcritical inviscid BVI due to the vortex acceleration. When the flow becomes supercritical, then the inviscid $C_{d}$ is higher because of the formation of supersonic pockets. This was verified by running the code for subcritical and supercritical cases. $C_{d}$ can be easily calculated in terms of the pressure distribution. Fig. 11 shows $C_{d}$ (T) for types $A, B$ and $C$. The $C_{d}$ signal seems to catch some part of the second disturbance whereas $C_{1}$ does not. This was also detected by Liu et al. [24] for some BVI cases using a thin layer Navier Stokes code, but is easier to see in the case of an oscillating flap [15], because there the periodicity of the motion 
is well-defined. Thus, we can conclude that the second disturbance is most probably related to the fluctuating $\mathrm{C}_{\mathrm{d}}$ caused mainly by the movement of the supersonic pocket.

In order to verify the above arguments we compared results from airfoils with the same thickness distribution (i. e. same family) but different camber, in conditions producing the same lift (i. e. different angle of attack). Fig. 12 compares the $C_{p}(T)$ BVI signals for the far-field (point $O$ ) for a NACA 1406 airfoil at an angle of attack $\alpha=0^{\circ}$, for a NACA $(0.5) 406$ at $\alpha=0.536^{\circ}$ and for a NACA 0006 at $\alpha=1.051^{\circ}$. These conditions, with a standing vortex upstream, produce initial $\mathrm{Cl}_{1}=0.229$. In fact, the entire unsteady lift $\mathrm{C}(\mathrm{T})$ for these cases is almost the same, whereas the unsteady drag $\mathrm{C}_{\mathrm{d}}(\mathrm{T})$ is not. All the airfoils have a type A shock motion for these conditions. We can see from Fig. 12 that disturbance I remains unchanged, whereas disturbance II does change, becoming higher as the camber increases. This agrees with our earlier discussion of lift and drag forces.

We also investigated the effect of the point of maximum camber. In Fig. 13 we compared the $\mathrm{C}_{\mathrm{p}}(\mathrm{T}) \mathrm{BVI}$ signals for the far-fieid (point $\mathrm{O}$ ) for NACA airfoils with different point of maximum camber: 1306 and 1406, for angles of attack that produce the same lift: $\alpha=0.055^{\circ}$ and $0^{\circ}$, respectively. We can see from Fig. 13 that disturbance I remains unchanged, whereas disturbance II becomes larger as the point of maximum camber moves downstream. Thus, the 1406 airfoil has the larger disturbance II.

From Figs 12 and 13 we can conclude that disturbance $I$ is related to the lift and disturbance II is related to the details of the airfoil shape, which produce a different supersonic pocket and thus a different drag. Symmetric aiffoils and airfoils with the point of maximum camber further upstream give a lower value of disturbance II, for the same lift.

A parametric study showed [26] that the vortex strength has a strong effect on the noise signal, whereas the vortex miss distance has only a weak effect. The maximum airfoil thickness and the details of airfoil shape were also found to be important. A more detailed parametric study is currently being carried out.

The directivity of the noise signal in the far-field is very complicated, as has been shown by experimental studies. Most of these experiments are also three-dimensional, which makes it very difficult to compare. For examp:e, in reference [42] it was shown for a model helicopter rotor that the maximum signal can have a different diection if the advance ratio $\mu$ is varied. 
Directivity is studied in a vortex-fixed coordinate system keeping the distance from the vortex $r_{V}$ constant $\left(r_{V}=50\right.$ chords). The relationship between the angles $\theta$ and $\theta_{V}$ in an airfoil fixed and a vortex fixed coordinate system is shown in Fig. 14. The $\mathrm{Cp}(\mathrm{t})$ signal for different directions is plotted in Figs $15,16,17$ for types $\mathrm{A}, \mathrm{B}$ and $\mathrm{C}$ shock motion, respectively (note that span $=4$ chords for this case). By inspecting the plots, we can see that disturbance $I$ is getting weaker as the direction angle $\theta$ increases from $0^{\circ}$ to $90^{\circ}$ (forward directivity). Disturbance II is getting stronger as the direction angle $\theta$ increases from $00^{\circ}$ to $90^{\circ}$ (downward directivity). The two disturbances also move closer as the angle $\theta$ is increased, and finally almost merge at $\theta=120^{\circ}$. This implies a different origin. If the origin of disturbance $\mathrm{I}$ is at the airfoil leading edge, the origin of disturbance II is probably somewhere downstream since this disturbance is related to the movement of the supersonic pocket.

- It should be noted that for an oscillating flap [15] the opposite directivity was observed: downward for disturbance I and forward for disturbance II. Thus, it was concluded that disturbance I is due to the dipole produced from oscillating lift and disturbance II is produced from oscillating drag. If we run a subcritical case then disturbance II disappears, as expected, and the directivity of disturbance I is downward (disturbance increases as $\theta$ is increased from $0^{\circ}$ to $90^{\circ}$ ). In both disturbances the directivity observed was not expected to be exactly the one described by a pure dipole, because of various nonlinearities (i. e. supersonic pocket) and source noncompactness. However, a main dipolelike behavior, as the one detected in the oscillating flap case [15], was expected. The reason that the directivity is different for an oscillating flap and BVI seems to be related to the difference in phase between the two disturbances. Disturbance II has a higher frequency than disturbance I and also a different phase with respect to disturbance I. Thus, it may add or subtract differently in different directions for various cases (i. e. diffraction). In the oscillating flap case the frequency and the phase difference are forced better than they are in the BVI case.

Consider next the noise signals resulting from types A, B and C shock motion (Figs 15, 16 and 17 , respectively). The two disturbances keep approaching each other as angle $\theta$ is increased because of the different disturbance origin. Angles $\theta=70^{\circ}$ and $60^{\circ}$, seem to give the maximum signal, if we measure from peak to peak, for types $A$ and $B$ respectively. (If we only look at the maximum absolute value then $\theta=0^{\circ}$ seems to produce the greatest noise). For the case of type $\mathrm{C}$ we can see that the entire 
region between $\theta=60^{\circ}$ and $\theta=90^{\circ}$ produces about the same noise. For subsonic cases (not shown) the maximum noise is $90^{\circ}$, as it was mentioned above.

Fig. 18 shows the directivity of the BVI for a NACA 0006 airfoil. In this case the two disturbances are closer to each other than the previous NACA 64 A006 cases. If we increase $\theta$, the two disturbances now fall on top of each other more quickly $\left(\theta=90^{\circ}\right)$. The noise becomes maximum at $\theta=90^{\circ}$, but still the noise at $\theta=60^{\circ}$ and $70^{\circ}$ is not much lower. Similar observations can be made for all the other 4-digit airfoils tried (e. g. 1306, 1406, (0.5)406).

From the preceding discussion of BVI noise directivity we can conclude that the maximum noise occurs at around $\theta=60^{\circ}-90^{\circ}\left(\theta_{V}=10^{\circ}-30^{\circ}\right)$, and depends on both disturbances I and II, but also on the phase difference between the two disturbances, that can be different for various airfoil shapes.

In order to isolate the effect of disturbance II in the maximum noise we compared (Fig. 19) the $\mathrm{Cp}(\mathrm{T})$ signal at the same point $\left(\theta=90^{\circ}\right.$ and $\mathrm{r}_{\mathrm{v}}=50$ chords $)$ for NACA airfoils $0006,(0.5) 406$ and 1406 for the conditions specified before for Fig. 12 (i. e. same lift). It can be noted that the two disturbances are almost merged at this point. We can also see that the NACA 1406 airfoil has a larger signal, which is consistent with the fact that the same airfoil has a larger disturbance II as shown in Fig. 12.

In Fig. 20 we compared the signal for NACA airfoils with different points of maximum camber: 1306 and 1406, for angles of attack that produce the same lift: $\alpha=0.055^{\circ}$ and $0^{\circ}$, respectively. We looked at the point of maximum noise: $\theta=90^{\circ}$ and $r_{V}=50$ chords. We can see that the 1406 airfoil has the larger signal, as expected since the same airfoil has a larger disturbance II as shown in Fig. 13.

We also ran the previously used 4-digit airfoils at subsonic Mach numbers at conditions producing the same lift. They all produced the same BVI disturbance at all angles, as expected (no disturbance II was observed). In conclusion, symmetric airfoils and airfoils with a point of maximum camber farther upstream seem to give less BVI noise for transonic conditions producing the same lift.

A direct comparison of the results obtained from the different methods (from small disturbance to Navier Stokes equations) shows that the results are very similar [7]. In fact, the further away we move from the airfoil surface the results tend to coincide. Thus, we expect that our conclusions will also hold if more accurate Euler/Navier-Stokes predictions are used. Three-dimensionality will, of course, 
influence the results. Some of the presented results will hold for three-dimensional cases, but only actual three-dimensional calculations (e. g. [43]) can show that.

\section{Conclusions}

An existing numerical finite difference code VTRAN2 was modified to analyze noise due to transonic BVI. The two-dimensional unsteady transonic small disturbance equation was solved numerically using ADI techniques with monotone switches, including viscous effects due to shockboundary interaction and the cloud-in-cell method for the simulation of the vortex. The Kirchhoff's method was used to extend the numerically calculated two-dimensional near-field aerodynamic results to the three-dimensional linear acoustic far-field.

The viscous effect (shock/boundary layer interaction) on BVI noise was studied and was found to have only a weak influence. The effect of the three types of unsteady shock motion ( $A, B$ and $C$ ) was also investigated. The unsteady pressure coefficients $C_{p}(t)$ showed the existence of two main disturbances. The first one (I) is believed to be associated with the fluctuating lift coefficient (Cl) and has a strong forward directivity while the second (II) is believed to be associated with the fluctuating drag coefficient $\left(C_{d}\right)$ caused by the movement of the supersonic pocket and has a strong downward directivity. The maximum radiation occurs at an angle $\theta$ between $60^{\circ}$ and $90^{\circ}$ below the horizontal for an airfoil-fixed coordinate system and depends on both disturbances I and II and the details of the airfoil shape. Symmetric airfoils, and airfoils with a point of maximum camber further upstream seem to give less BVI noise for conditions producing the same lift.

It is hoped that this work can contribute toward the better understanding of the mechanisms of noise due to transonic BVI. In the future, we plan to study the influence of the details of airfoil shape, especially near the leading edge. We will also include the effects of an oscillating airfoil at the same time with a BVI that can be useful in actual helicopter cases (feathering).

\section{Acknowledgements}

The authors want to thank Dr. P. M. Goorjian from NASA Ames Research Center for providing the new version of LTRAN2. The calculations were performed at the computational facilities of Minnes rta Supercomputer Institute (MSI) and at the Cornell Universiry Production Supercomputer Facility of the Center for Theory and Simulation in Science and Engineering. Finally, this research was 
supported by a Grant-in-aid of Research from the Graduate School of the University of Minnesota, and NASA grants NAG 2-588 and NAG 2-646. C. Kitaplioglu was the technical monitor and his suggestions during the course of the research are greatly appreciated by the authors.

\section{References}

1. George, A. R., "Helicopter Noise: State-of- the-Art," Journal Aircraft, Vol. 15, No. 11, November 1978, pp. 707-715.

2. Schlinker, R. H., and Amiet, R. K., "Rotor-Vortex Interaction Noise," NASA CR-3744, Oct. 1983.

3. Schmitz, F. H., and Yu, Y. H., "Helicopter Impulsive Noise: Theoretical and Experimental Status," in Recent Advances in Aeroacoustics, A. Krothapalli and C. A. Smith, editors, Springer-Verlag, NY, 1986.

4. George, A. R., Smith C. A., Maisel, M. D., and Brieger, J. T., "Tilt Rotor Aircraft Aeroacoustics,"Proceedings of the 45 th Annual Forum of the American Helicopter Society, Boston, MA., May 1989.

5. Poling, D. R., Dadone, L., Telionis, D. P., "Blade-Vortex Interaction," AIAA Journal, Vol. 27, No. 6, June 1989, pp. 694-699.

6. George, A. R., and Chang, S. B., "Noise Due to Transonic Blade-Vortex Interactions," Proceedings of the 39th Annual National Forum of the American Helicopter Society, Paper A83-39-50-D000, 1983.

7. George, A. R., and Chang, S. B., "Flow Field and Acoustics of Two-dimensional Transonic Blade-Vortex Interactions," AIAA Paper 84-2309, AIAA 9th Aeroacoustics Conference, Williamsburg, VA, October 15-17, 1984.

8. Ballhaus, W. F., and Goorjian, P. M., "Implicit Finite-Difference Computations of Unsteady Transonic Flows about Airfoil," AIAA Journal., Vol. 15, No. 12, Dec. 1977, pp. 1728-1735.

9. Rizzetta D. P., and Chin, W. C., "Effect of Frequency in Unsteady Transonic Flow," AIAA Journal, Vol. 17, No. 7, July 1979, pp. 779-781.

10. Guruswamy P., and Goorjian P. M., "Effects of Viscosity on Transonic Aerodynamic and Aeroelastic Characteristics of Oscillating Airfoils" AIAA Journal, Vol 21, No. 9, Sept. 1984, pp.700-707.

11. Goorjian, P. M., Meagher, M. E., and Van Buskirk, R. D., "Monotone Switches in Implicit Algorithms for Potential Equations Applied to Transonic Flows" AIAA Journal, Vol 23, No. 4, Apr. 1985, pp. 492-498.

12. Goorjian, P. M., and Van Buskirk, R. D., "Second-Order-Accurate Spatial Differencing for the Transonic Small-Disturbance Equation" AIAA Journal, Vol. 23, No. 11, Nov. 1985, pp. 16931699.

13. Lyrintzis, A. S., and George, A. R., "VTRAN2: Computation of Two-dimensional Blade-Vortex Interactions Using the Unsteady Small Disturbance Equation, (User's Manual)" Cornell Liniversity, Ithaca NY, March 1986. 
14. Lyrintzis, A. S. "Transonic Blade-Vortex Interactions," Ph.D. Dissertation, Mechanical and Aerospace Engineering Department, Cornell University, Ithaca NY, Jan. 1988.

15. Lyrintzis, A. S., and Xue, Y., "Acoustics of Transonic Flow Around an Oscillating Flap," submitted in the Journal of Fluids Engineering for publication, June 1990.

16. McCroskey, W. J., and Goorjian, P. M., "Interactions of Aiffoils with Gusts and Concentrated Vortices in Unsteady Transonic Flow," AIAA Paper 83-1691, AIAA 16th Fluid and Plasma Dynamics Conference, Danvers, MA, July 1983.

17. Srinivasan, G. R., McCroskey, W. J., and Kutler, P., "Numerical Simulation of the Interaction of a Vortex with Stationary Airfoil in Transonic Flow," AIAA Paper 84-0254, AIAA 22nd Aerospace Sciences Meeting, Reno, Nevada, Jan., 1984.

18. Sankar, N. L., and Tang, W., "Numerical Solution of Unsteady Viscous Flow Past Rotor Sections," ÁIAA Paper 85-129, Jan. 1985.

19. Srinivasan, G. R., McCroskey, W. J., and Baeder J. D., "Aerodynamics of two-dimensional Blade-Vortex Interaction," AIAA Journal, Vol. 24 No. 10, Oct. 1986, pp. 1569-1576.

20. Owen S. T., and Shenoy R. K., "Numerical Investigation of Two-Dimensional Blade-Vortex Interaction," . Proceedings of the AHS National Specialists' Meeting on Aerodynamics and Aeroacoustics, Arlington, Texas, Feb. 25-27, 1987.

21. Damodaran, M., and Caughey, D. A., "A Finite Volume Euler Calculation of the Aerodynamics of Transonic Airfoil-Vortex Interaction," AIAA Journal, Vol. 26, No. 11, Nov. 1988, pp.13461353.

22. Baeder, J. D., McCroskey, W. J., and Srinivasan, G. R.,"Acoustic Propagation Using Computational Fluid Dynamics," Proceedings of the 42 nd Annual Forum of the American Helicopter Society, Washington, DC, June, 1986, Vol. 1, pp. 551-562.

23. Baeder, J. D., "Computation of Non-Linear Acoustics in Two-Dimensional Blade-Vortex Interactions,". 13th European Rotorcraft Forum, Arles, France, Sept. 1987.

24. Liu, N. S. Davoudzadeh, F., Briley, W. R., and Shamroth, S. J., "Navier Stokes Simulation of Transonic Blade-Vortex Interactions," Proceedings of the International Symposium on Nonsteady Fluid Dynarics (ASME), Miller, J. A., and Telionis, D. P., editors, Toronto, Canada, June 1990, pp 177-186.

25. George, A. R., and Lyrintzis, A. S., "Acoustics of Transonic Blade-Vortex Interactions," AIAA Journal, Vol. 26, No. 7, July 1988, pp. 769-776.

26. Lyrintzis, A. S., and George, A. R., "Far-Field Noise of Transonic Blade-Vortex Interactions," American Helicopter Society Journal, Vol. 34, No.3, July 1989, pp.30-39.

27. Lyrintzis, A. S., and George, A. R., "The Use of Kirchhoff Method in Acoustics," AIAA Journal, Vol. 27, No. 10, Oct. 1989, pp. 1451-1453.

28. Lyrintzis, A. S., and George, A. R., "Study of Transonic Blade-Vortex Interaction Noise,"Noise Control Engineering Journal, Vol. 32. No. 3, May-June 1989, pp.105-109.

29. Lyrintzis, A. S., and Xue, Y., "Noise Mechanisms of Transonic Blade-Vortex Interactions" Proceedings of the American Helicopter Society 46th Annual Forum, Washington, DC, May 1990. 
30. Ballhaus, W. F., "Some Recent Progress in Transonic Flow Computations," in Numerical Methods in Fluid Dynamics, ed. Wirz H. J., and Smolderen J. J., McGraw Hill, NY 1978, pp. 155-235.

31. Poling, D. R., Telionis, D. P., "The Response of Airfoils to Periodic Disturbances-The Unsteady Kutta Condition," AIAA Journal, Vol.24, No.2, Feb. 1986, pp. 193-199.

32. Chang, S. B., "Aerodynamics and Acoustics of Transonic Two-Dimensional Blade-Vortex Interactions," Ph.D. Dissertation, Mechanical and Aerospace Engineering Department, Cornell University, Ithaca NY, Jan. 1985.

33. Rizzetta, D. P., "Procedures for the Computation of Unsteady Transonic Flow Including Viscous Effects," NASA CR-166249, 1982.

34. Morgans, R. P. "The Kirchhoff Formula Extended to a Moving Surface," Philosophical Magazine, 9 (s.7, No. 55), 1930, pp. 141-161.

35. Morino, L., "A General Theory of Unsteady Compressible Potential Aerodynamics," NASA Contractor Report CR-2464, December 1974.

36. Morino, L., "Steady, Oscillatory, and Unsteady Subsonic and Supersonic Aerodynamics Production Version 1.1 (SOUSSA-P, 1.1), Vol. 1, Theoretical Manual," NASA Contractor

- Report CR-159130, 1980.

37. Farassat, F., and Myers M. K., "Extension of Kirchhoff's formula to Radiation from Moving Surfaces," Journal of Sound and Vibration, Vol. 123, No. 3, 1988, pp.451-460.

38. Tijdeman, H., "Investigations of the Transonic Flow Around Oscillating Airfoils," NLR TR 77090- U, NLR The Netherlands, 1977.

39. Tangler, J. L., "Schlieren and Noise Studies of Rotors in Forward Flight," Paper 77, 33-05, Presented at the 33rd Annual National Forum of the American Helicopter Society, Washington, D. C., May 1977.

40. Caradonna, F. X., Laub, G. H., and Tung, C., "An Experimental investigation of the Parallel Blade-Vortex Interaction," 10th European Rotorcraft Forum, The Hague, Netherlands, August 1984.

41. Shenoy, R. K. "Aeroacoustic Flowfield and Acoustics of a Model Helicopter Tail Rotor at a High Advance Ratio," Proceedings of the 45th Annual Forum of the American Helicopter Society, Boston, MA., May 1989.

42. Martin, M. R., and Splettstoesser, W. R., "Acoustic Results of the Blade-Vortex Interaction Acoustic Test of a 40 Percent Model Rotor in the DMW," Proceedings of the American Helicopter Society National Specialists' Meeting on Aerodynamics and Aeroacoustics, Arlington, Texas, Feb. 1987.

43. Tadghighi, H., Hassan, A. A., and Charles, B., "Prediction of Blade-Vortex Interaction Noise Using Airloads Generated by a Finite-Difference Technique," Proceedings of the 46th Annual Forum of the American Helicopter Society, Washington DC, May 1990, Vol. I, pp. 367-378. 


\section{Figure Captions}

Figure 1. Formulation of BVI for a four bladed rotor (from Schlinker and Amiet [2]).

Figure 2. Two-dimensional BVI.

Figure 3. Kirchhoff's surface for the calculation of the far-field.

Figure 4. Effect of $y$-grid size on the $\mathrm{Cl}(\mathrm{T})$ signal for type $\mathrm{C}$

Figure 5. Effect of viscosity on the $C_{p}(T)$ signal for type $A$; point $P(x=-0.3000, y=-0.17478)$

Figure 6. Effect of viscosity on the $\mathrm{C}(\mathrm{T})$ signal for type A;

Figure 7. Effect of viscosity on the $C_{d}(T)$ signal for type $A$;

Figure 8. Comparison of the near-field BVI noise for types A, B, C; point $P(x=-0.3000, y=-0.17478)$

Figure 9. Comparison of the far-field BVI noise for types A, B, C; point $O\left(r=20\right.$ chords, $\theta=30^{\circ}$, span $=8$ chords).

Figure 10. Comparison of the $\mathrm{Cl}$ (T) signal for BVI, types A, B, C.

Figure 11. Comparison of the $\mathrm{C}_{\mathrm{d}}(\mathrm{T})$ signal BVI for types $\mathrm{A}, \mathrm{B}, \mathrm{C}$.

Figure 12. Comparison of the far-field noise for NACA $0006\left(\alpha=1.051^{\circ}\right),(0.5) 406\left(\alpha=0.536^{\circ}\right)$ and $1406\left(\alpha=0^{\circ}\right)$, initial $C_{l}=0.229$ in all cases; foint $O\left(r=20\right.$ chords, $\theta=30^{\circ}$, span $=8$ chords $)$.

Figure 13. Comparison of the far-field noise for NACA $1306\left(\alpha=0.055^{\circ}\right)$ and $1406\left(\alpha=0^{\circ}\right)$, initial $C_{l}=$ 0.229 in both cases; point $\mathrm{O}\left(\mathrm{r}=20\right.$ chords, $\theta=30^{\circ}$, span $=8$ chords $)$.

Figure 14. Relation between an airfoil fixed and a vortex fixed coordinate system.

Figure 15 . BVI noise directivity for type $\mathrm{A} ; \mathrm{r}_{\mathrm{V}}=50$ chords, span $=4$ chords.

Figure 16 . BVI noise directivity for type $B ; r_{V}=50$ chords, span $=4$ chords.

Figure 17. BVI noise directivity for type $C ; \mathrm{r}_{\mathrm{v}}=50$ chords, span $=4$ chords.

Figure 18. BVI noise directivity for NACA 0006; $\mathrm{r}_{\mathrm{V}}=50$ chords, span $=4$ chords.

Figure 19. Comparison of the far-field noise for NACA $0006\left(\alpha=1.051^{\circ}\right),(0.5) 406\left(\alpha=0.536^{\circ}\right)$ and $1406\left(\alpha=0^{\circ}\right)$, initial $C_{l}=0.229$ in all cases; $\theta=90^{\circ}, r_{v}=50$ chords, span $=4$ chords.

Figure 20. Comparison of the far-field noise for NACA $1306\left(\alpha=0.055^{\circ}\right)$ and $1406\left(\alpha=0^{\circ}\right)$, initial $\mathrm{Cl}_{\mathrm{l}}=0.229$ in both cases; $\theta=90^{\circ}, \mathrm{r}_{\mathrm{V}}=50$ chords, span $=4$ chords. 


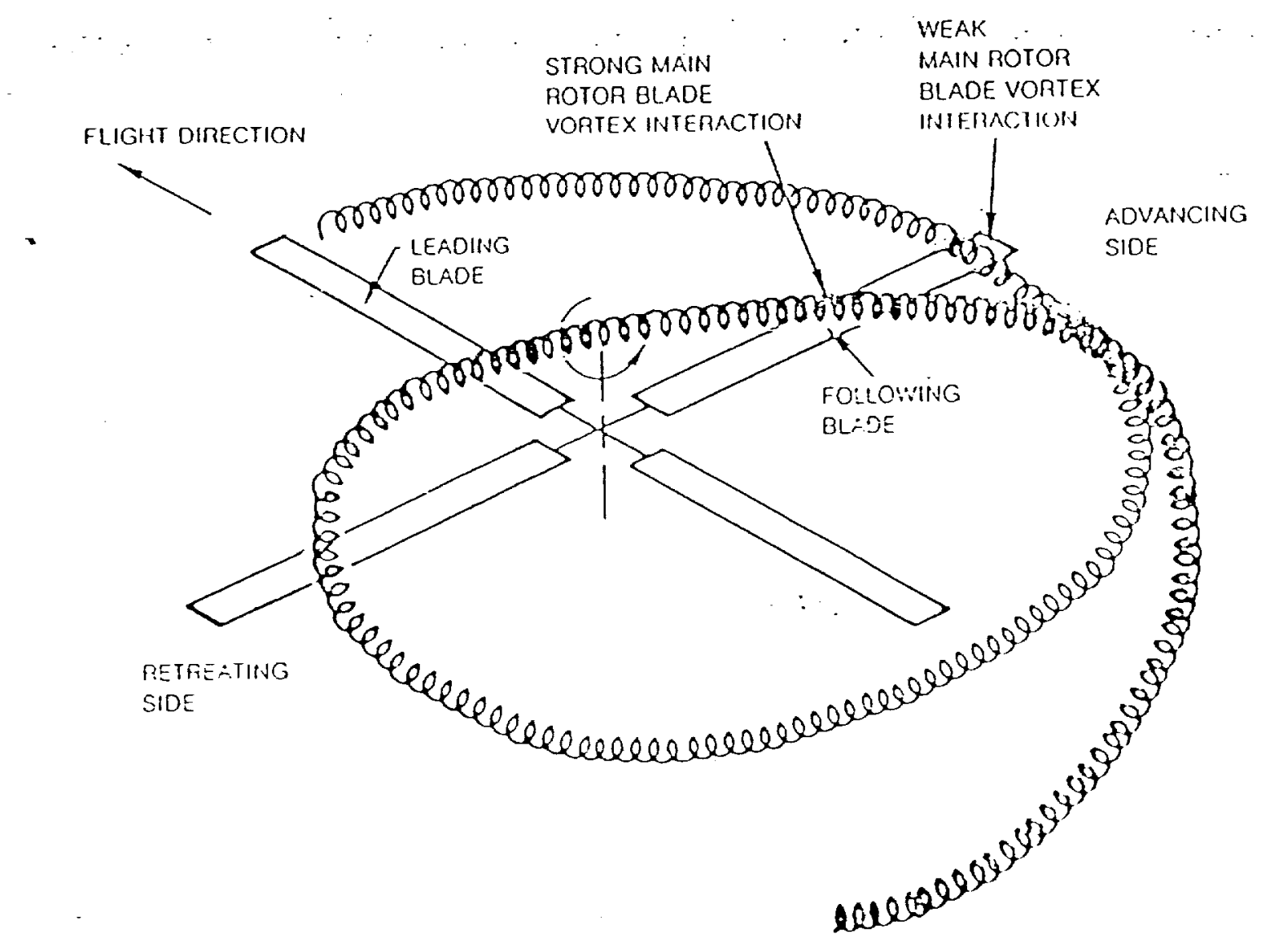



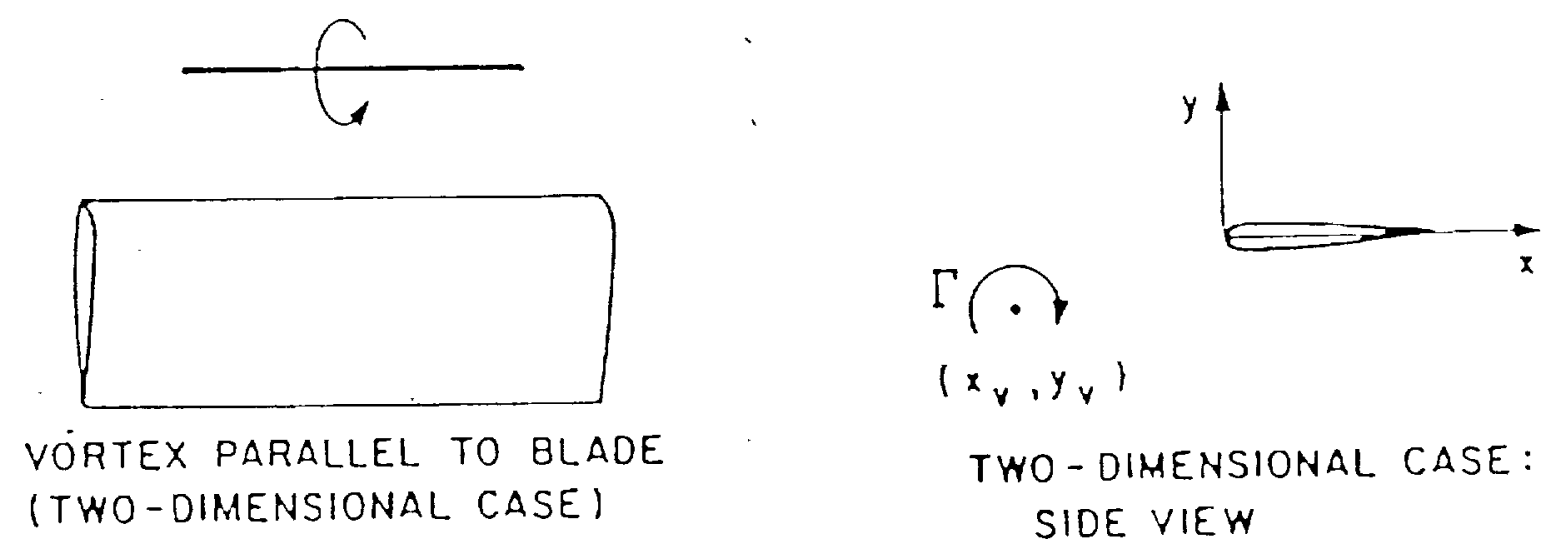
SIDE VIEW 
?
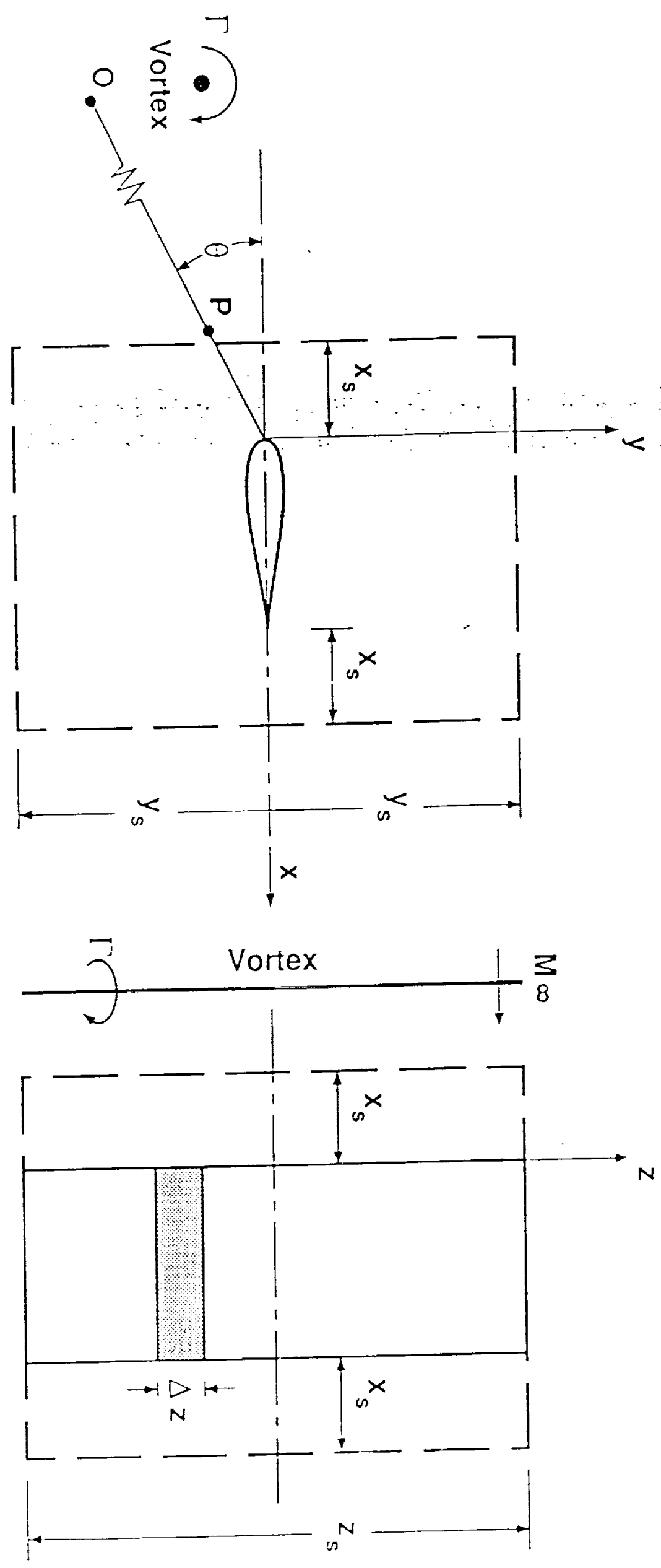

tig. 3 


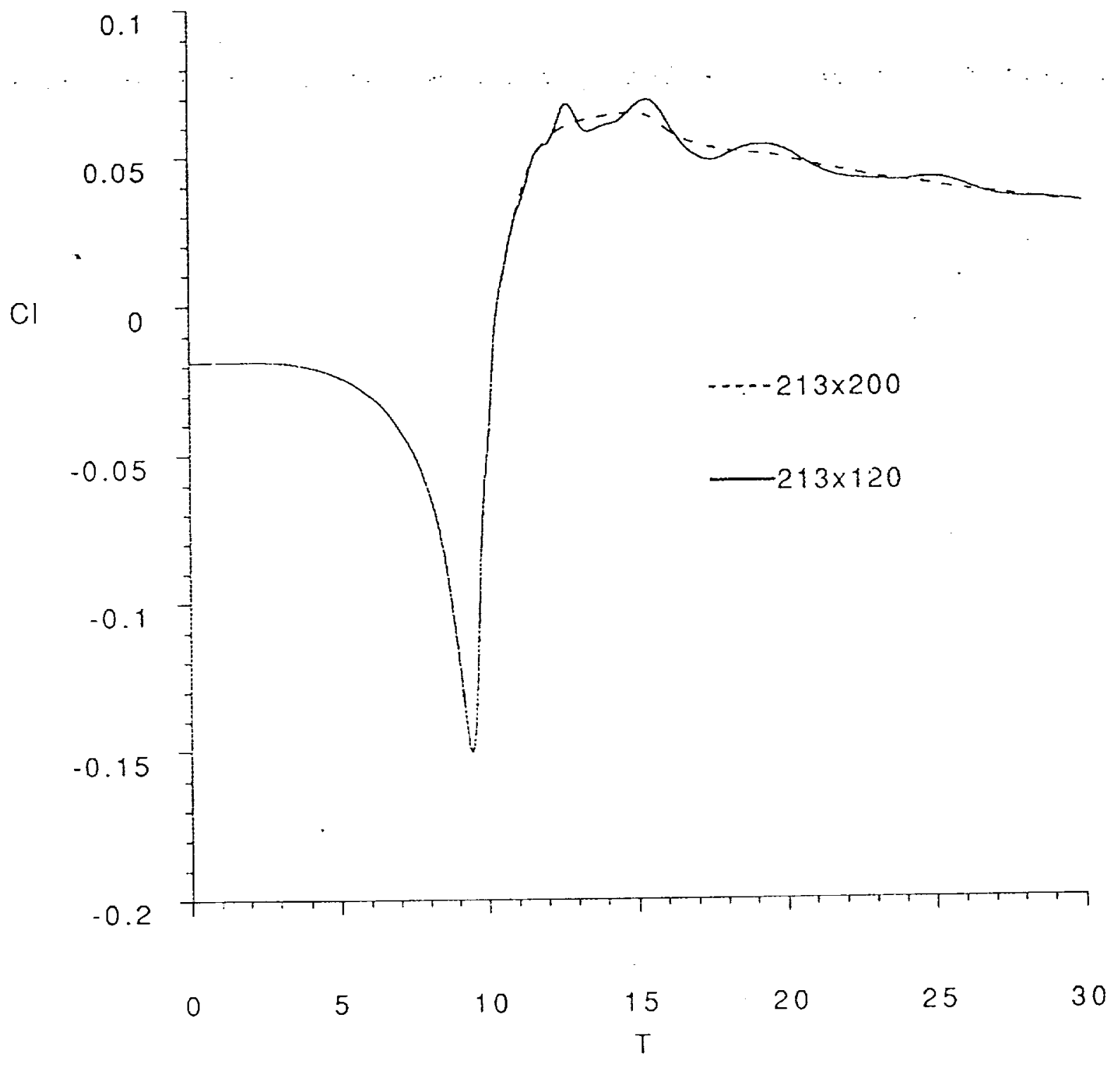

fig 4 


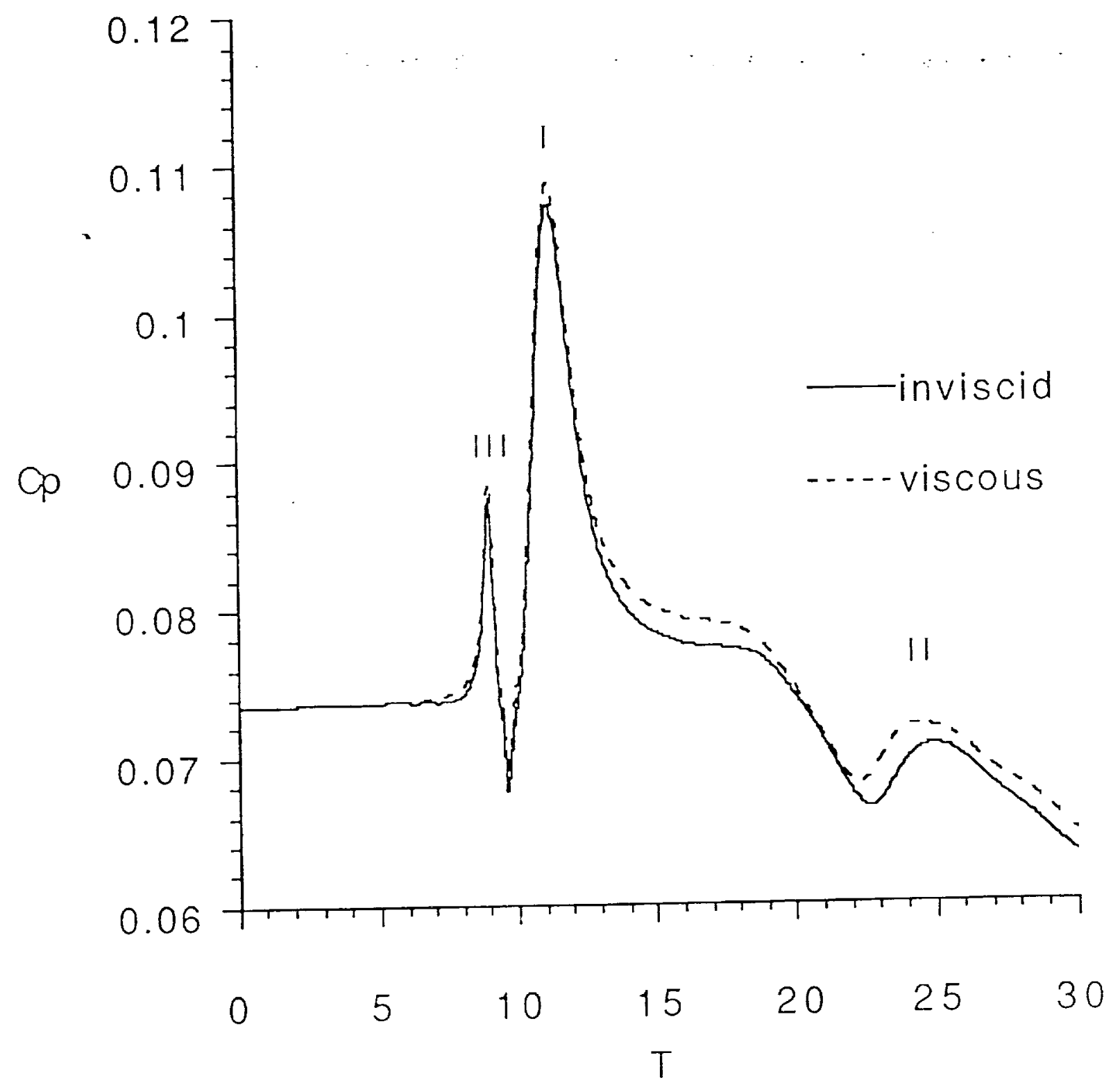

tigs 


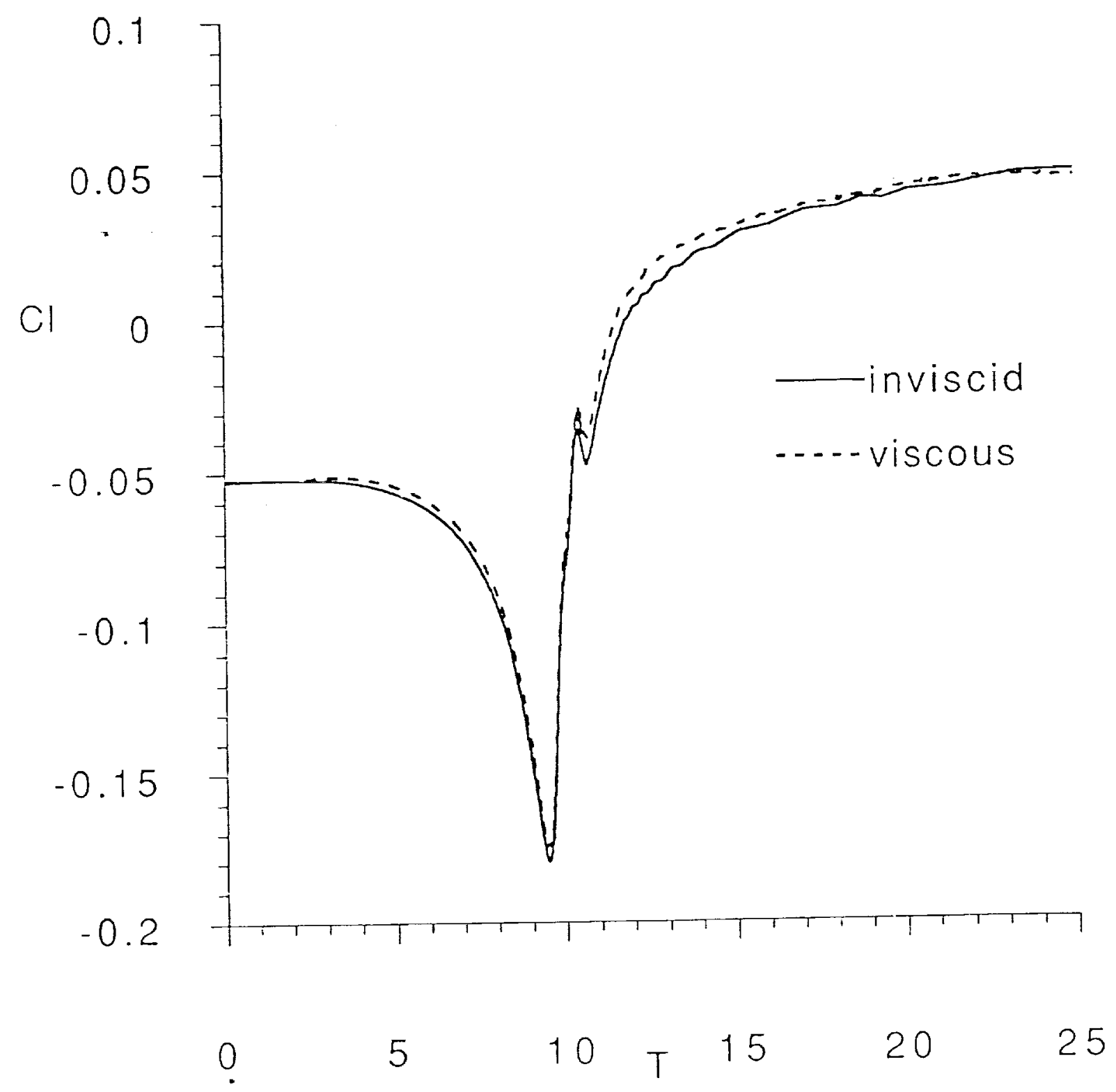




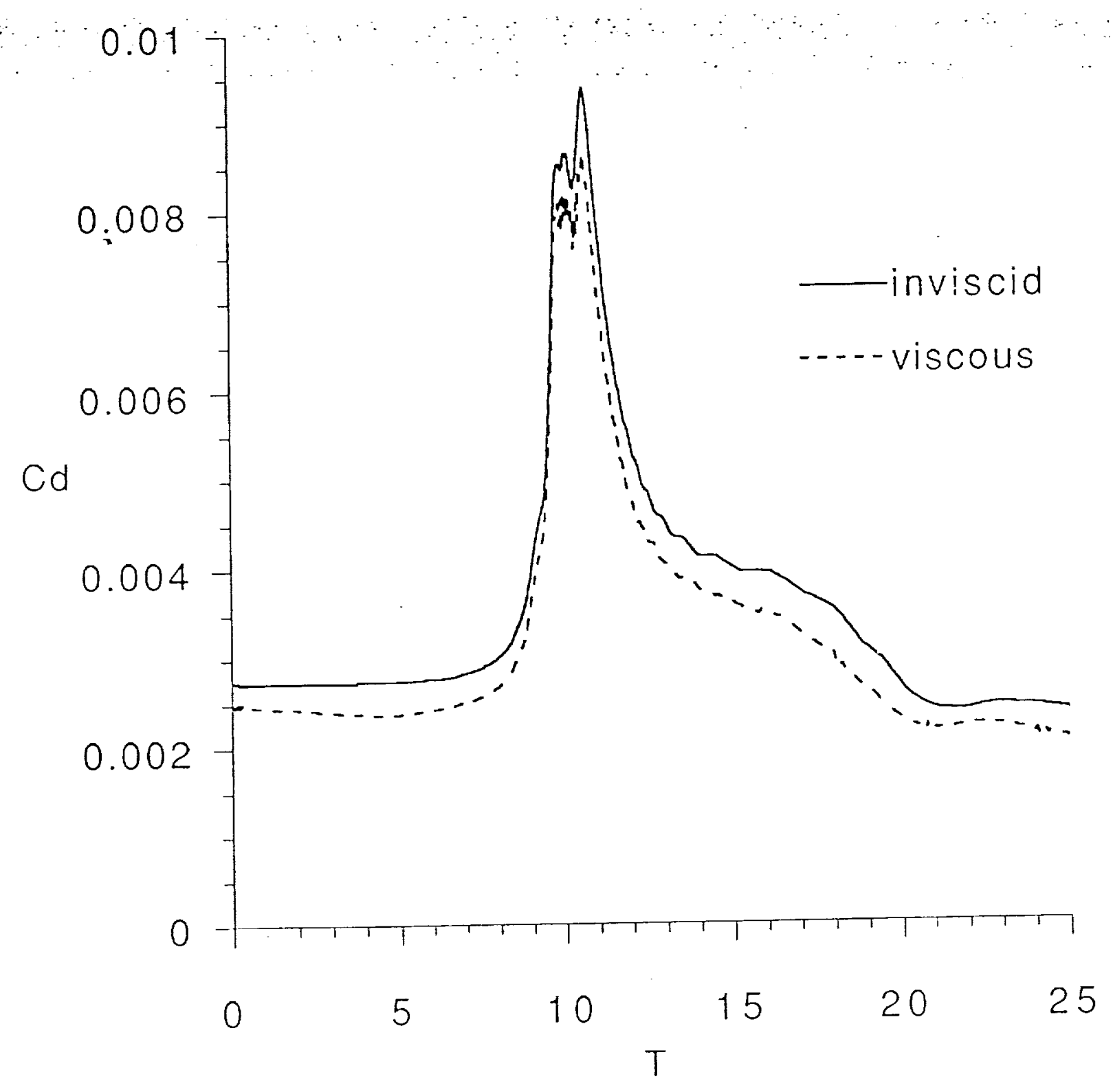

tig 7 


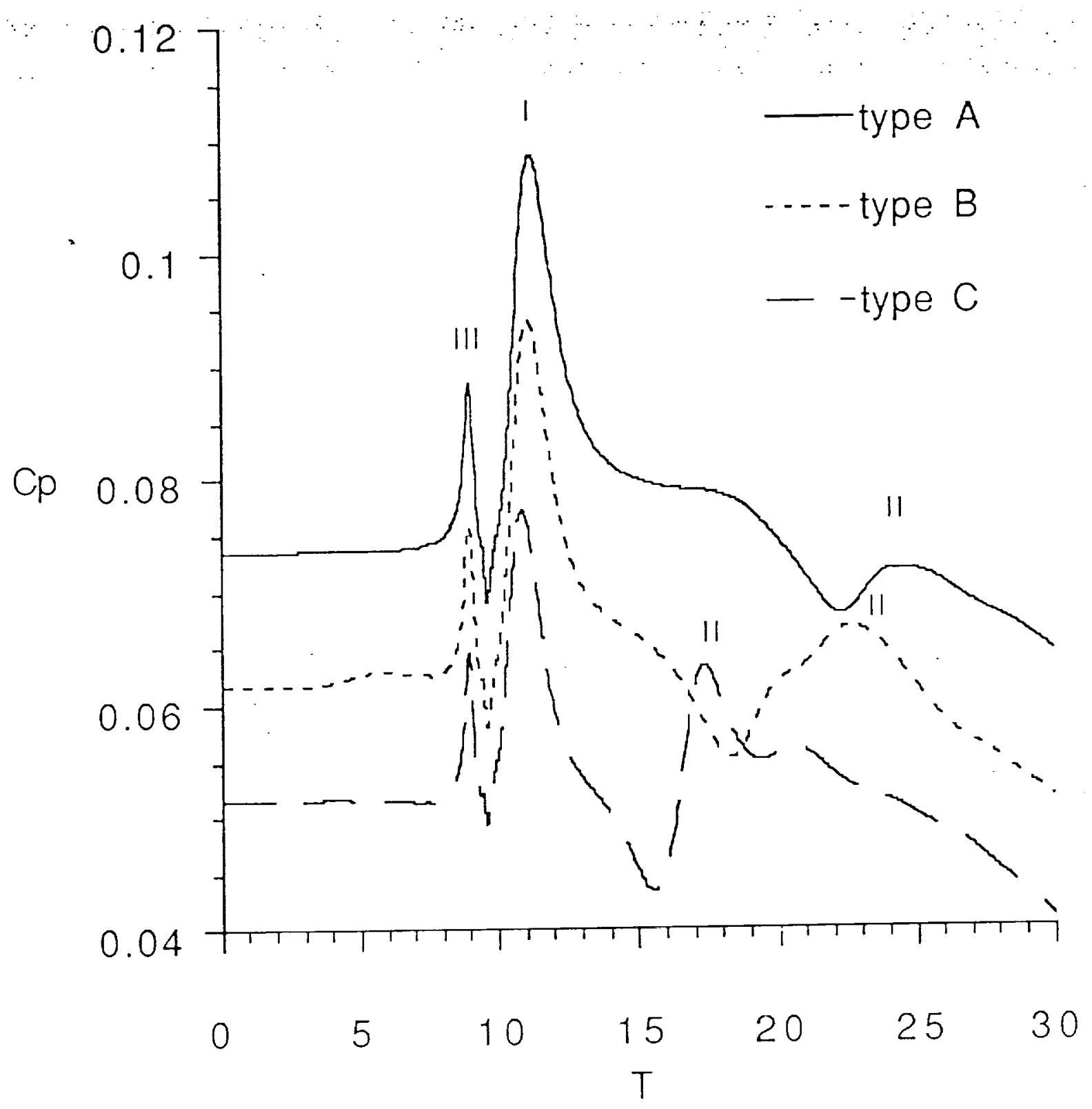




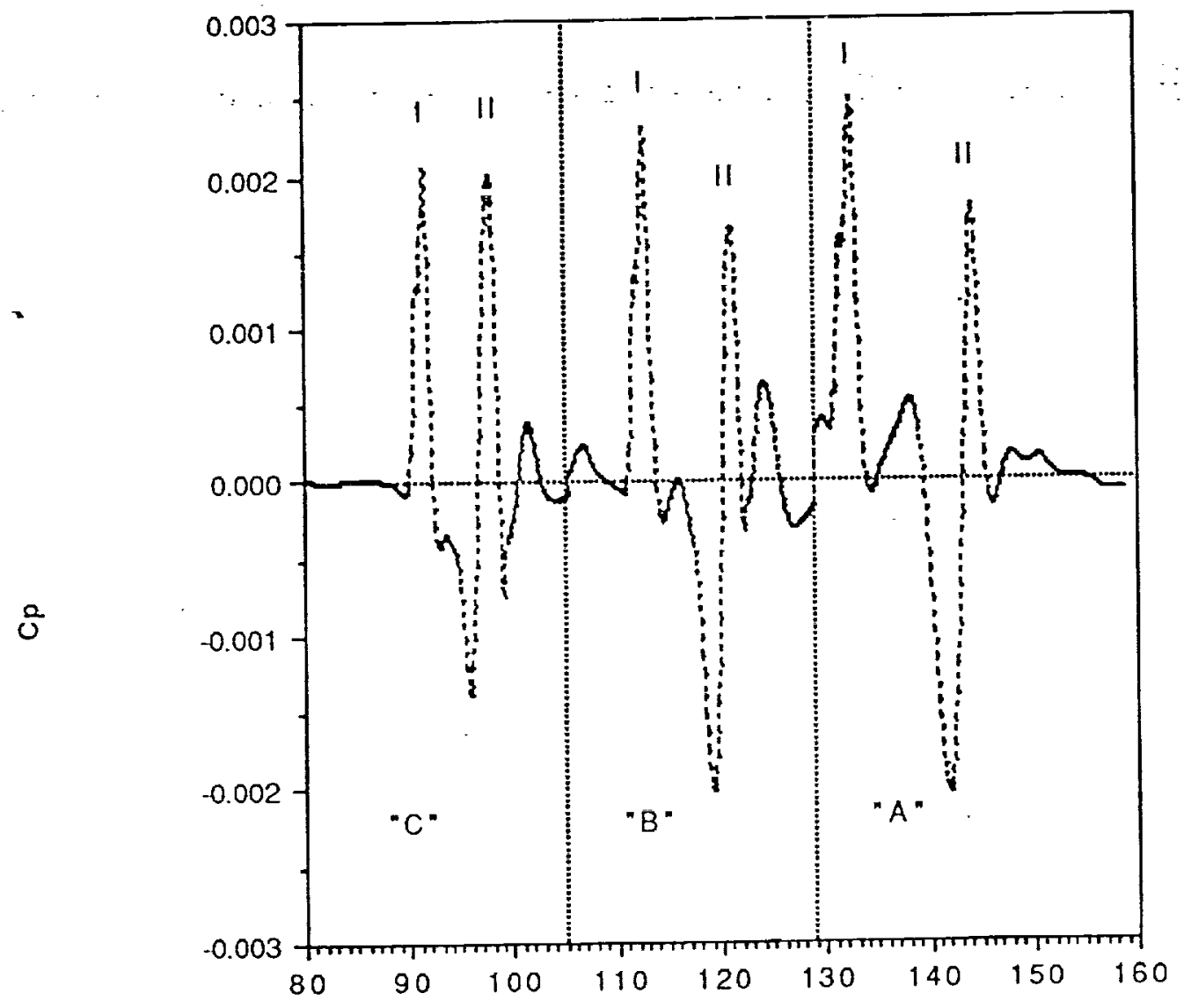

$T$ 


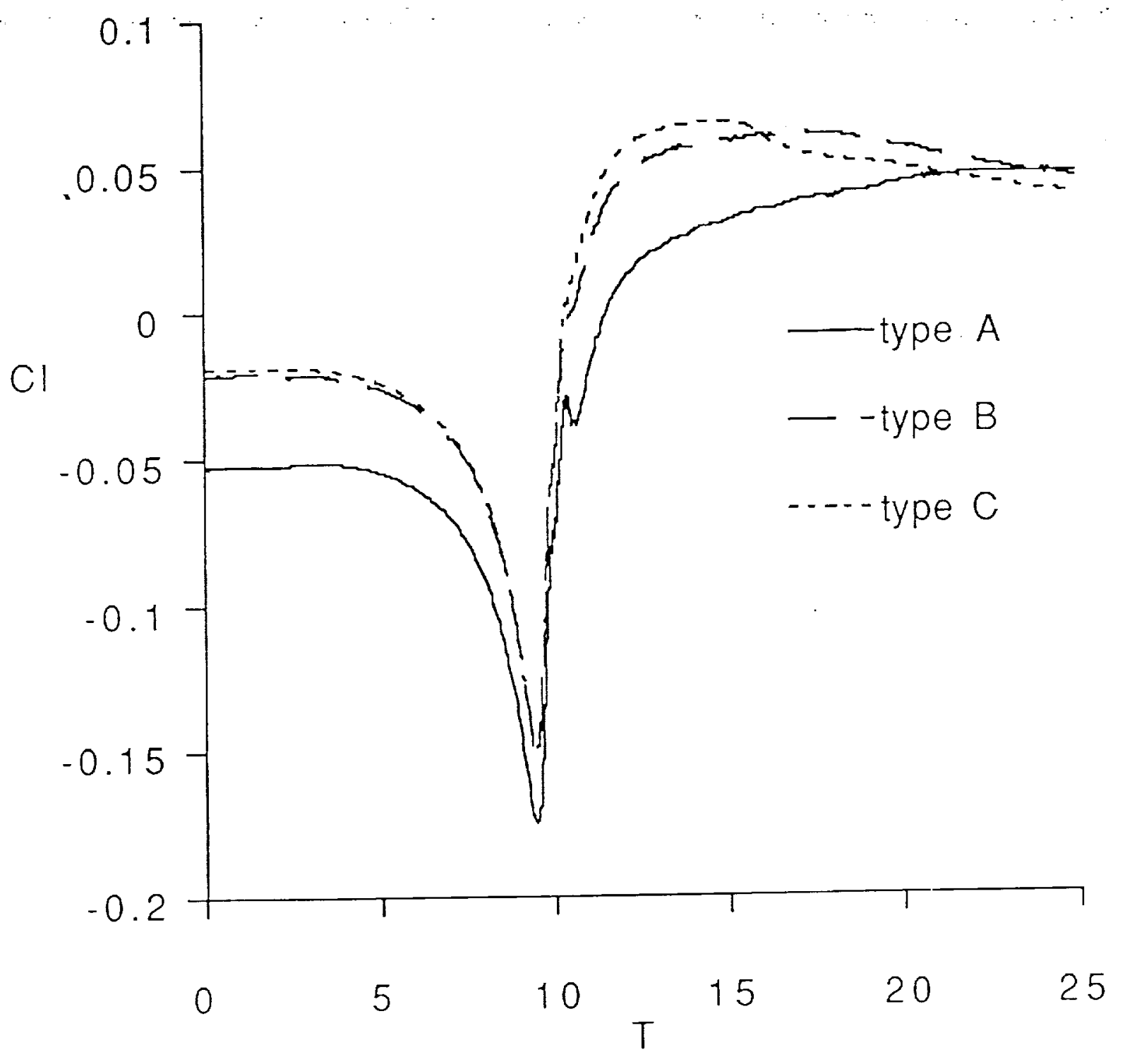

tig 10 


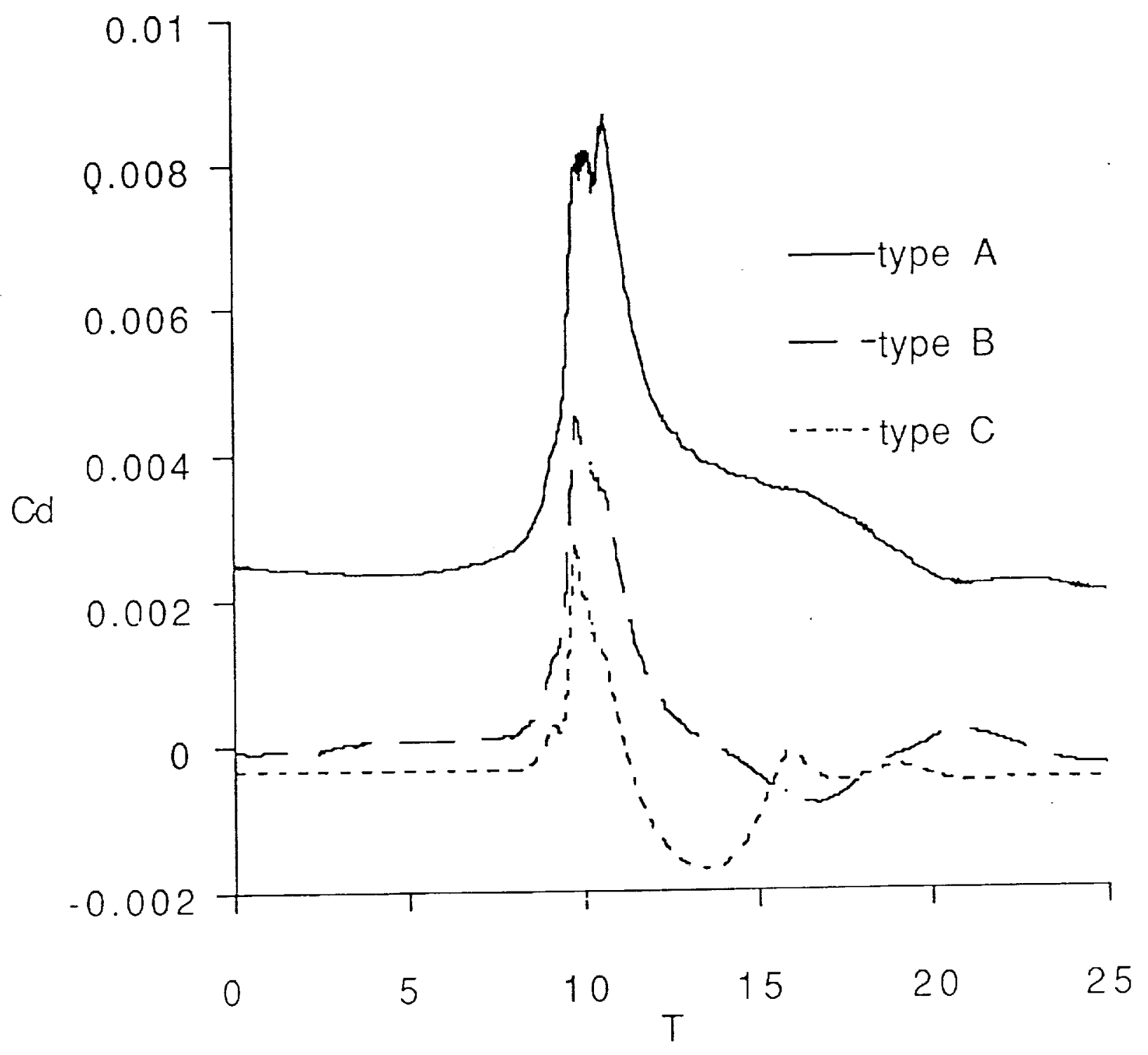




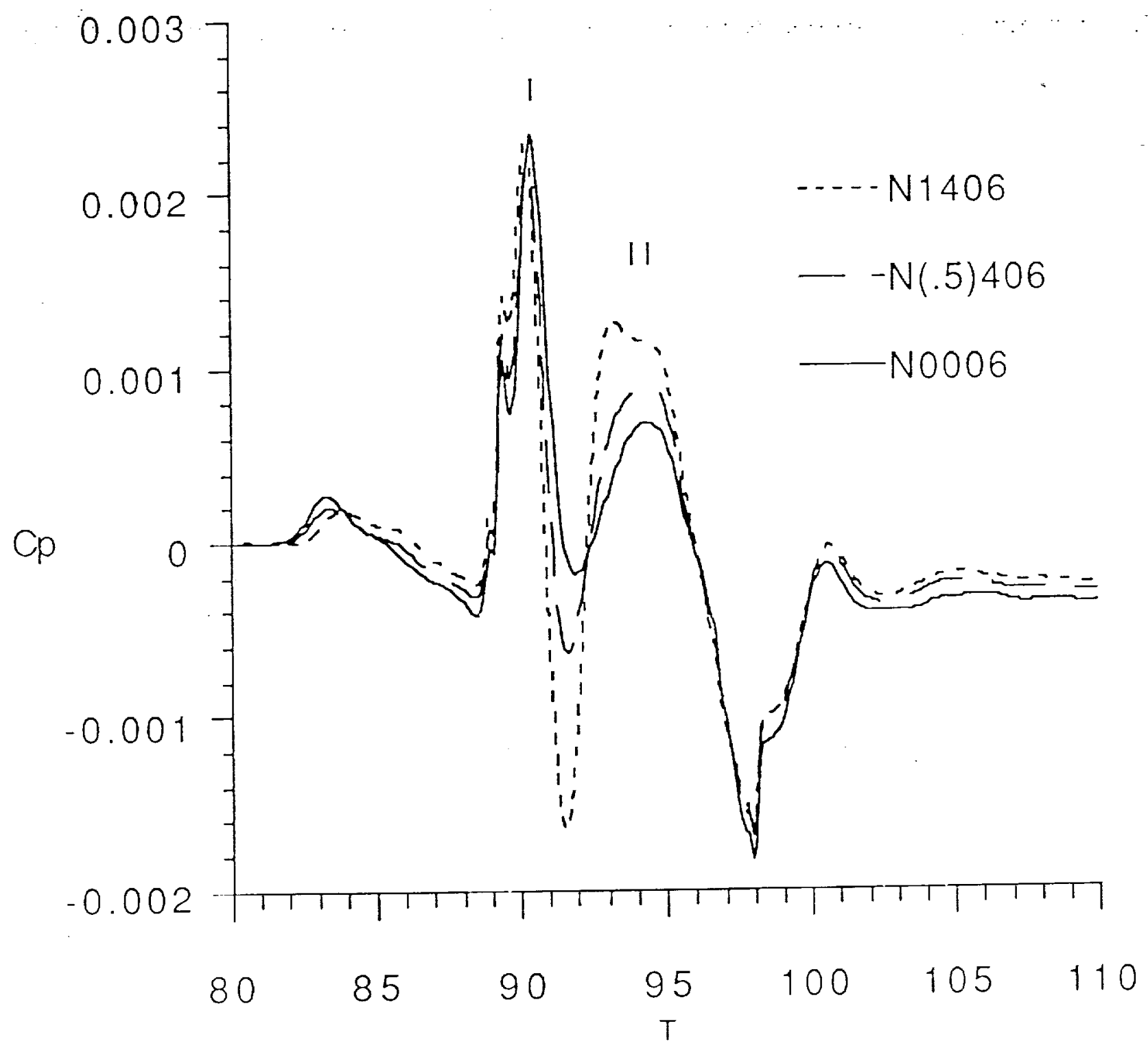




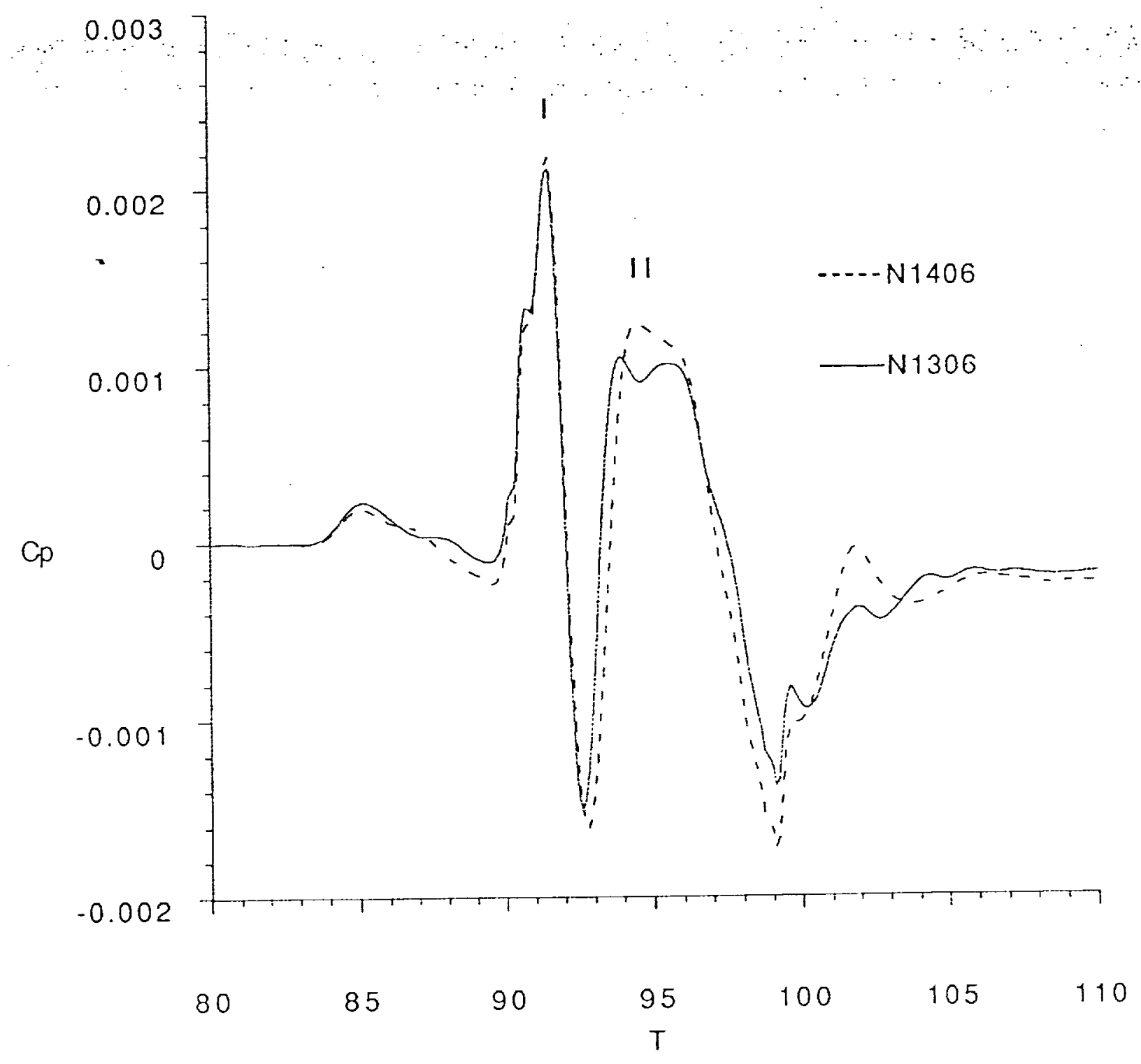

tig 13 


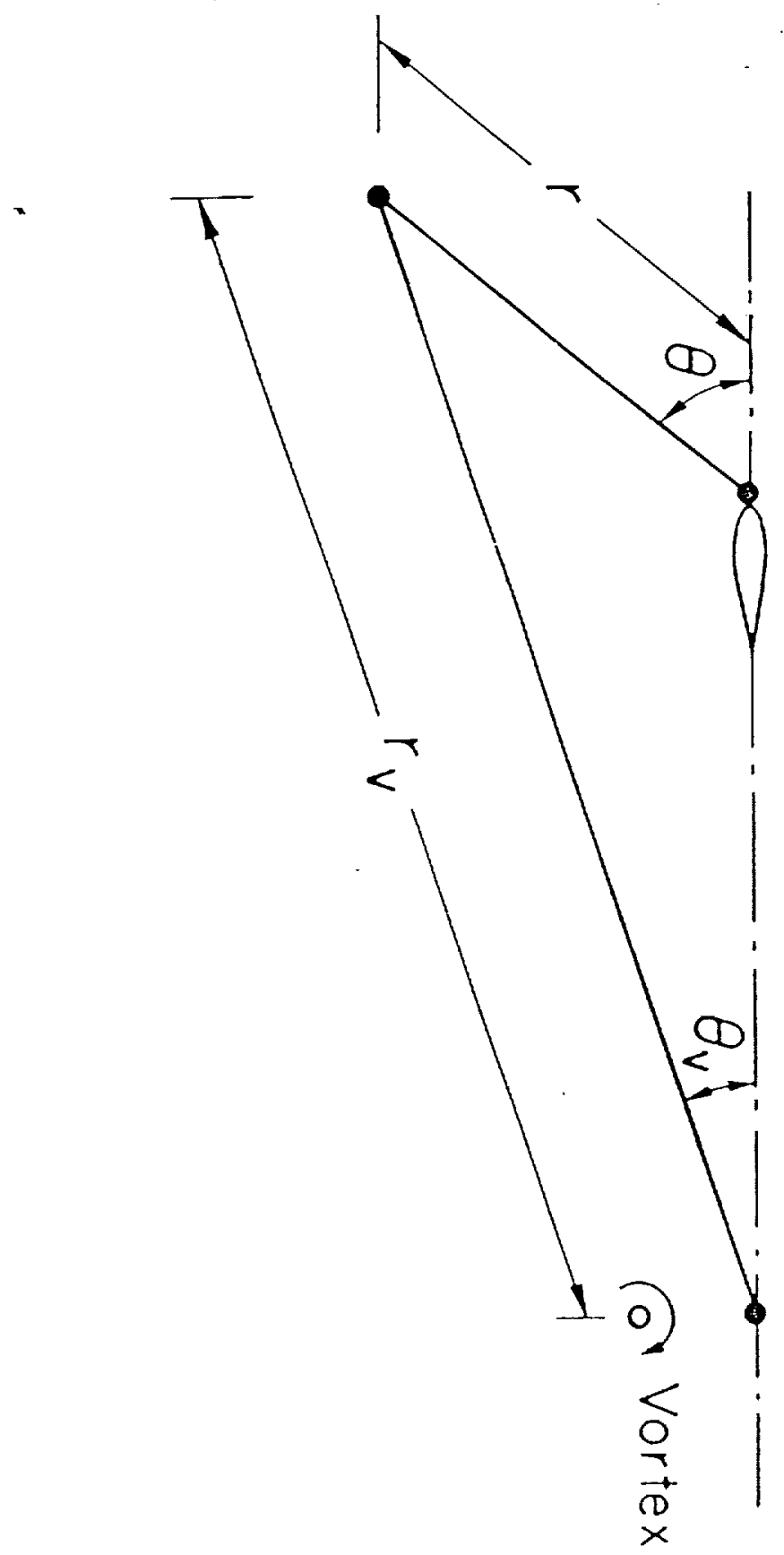

figl 14 


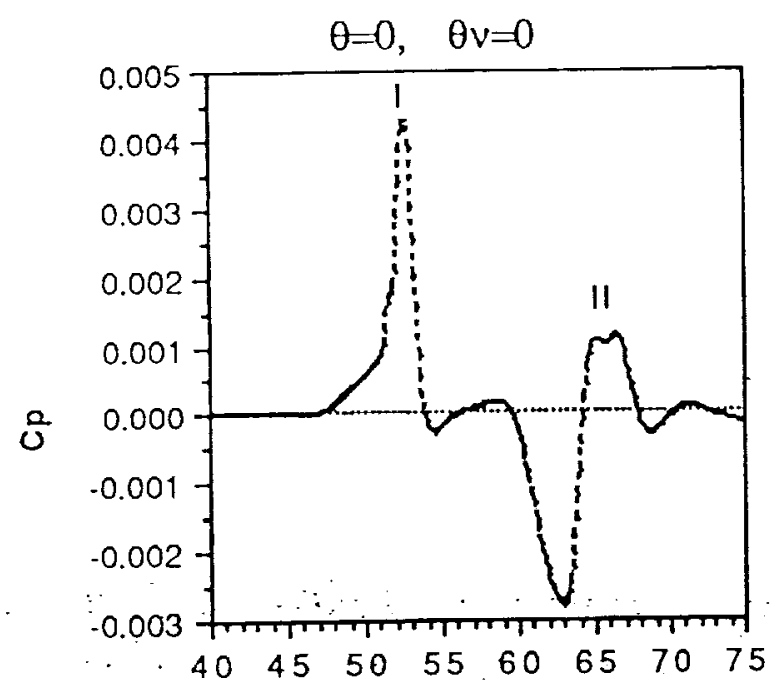

T

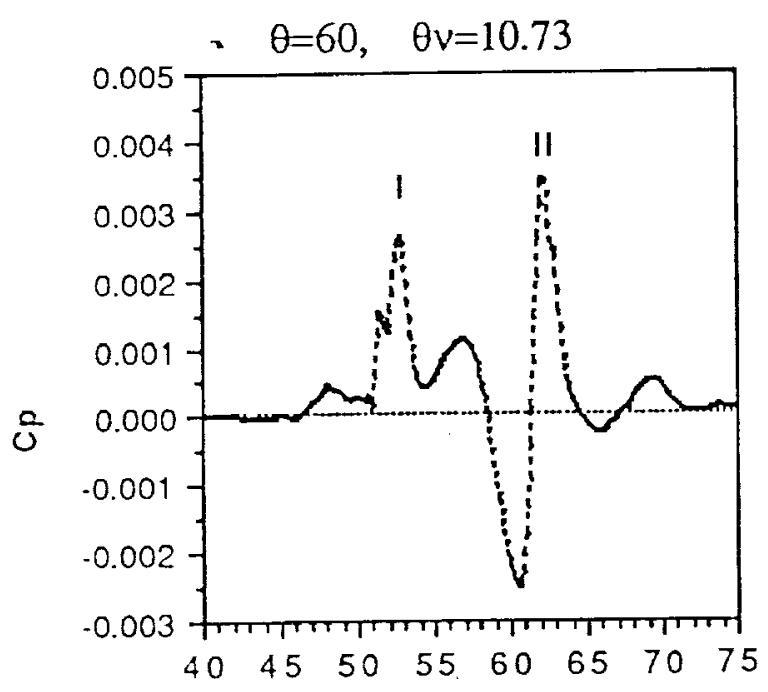

$T$

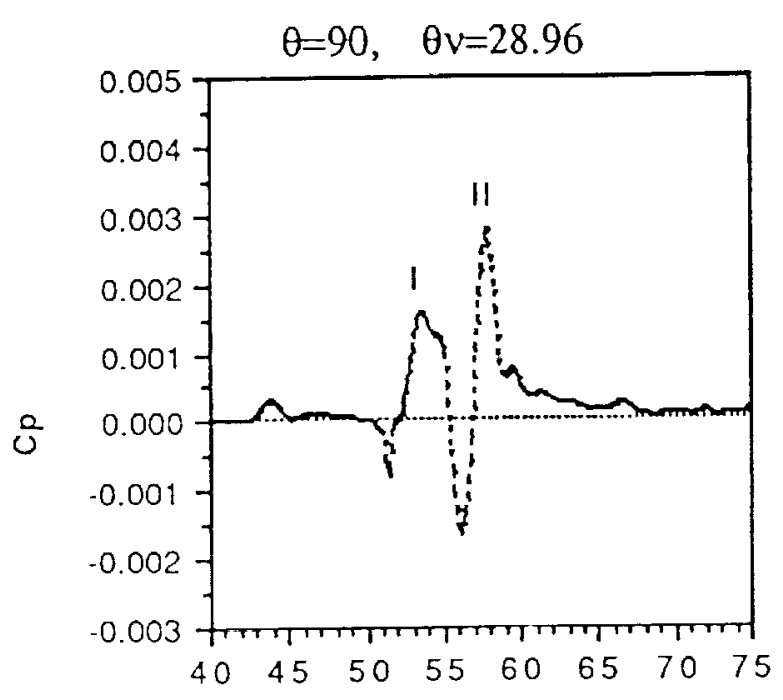

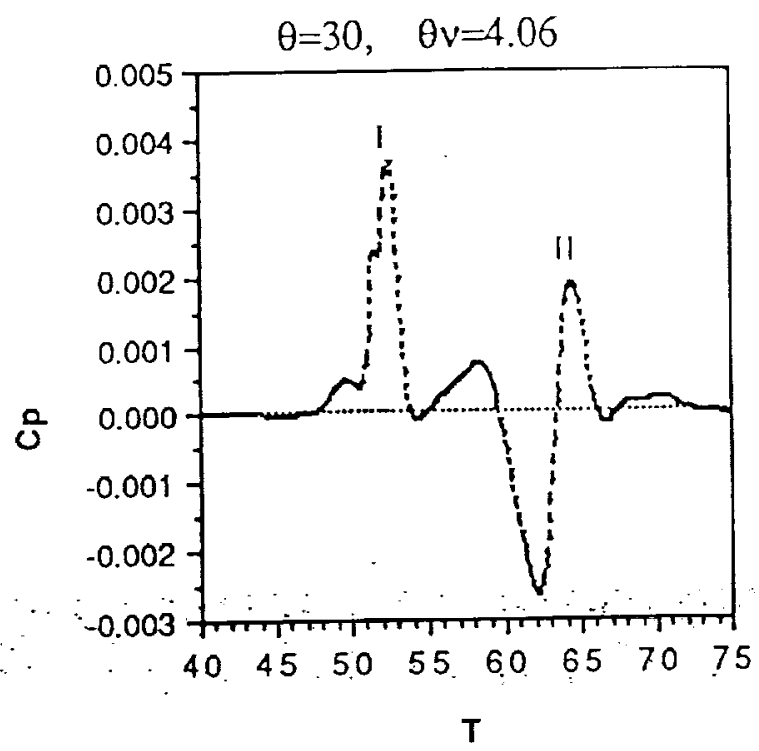
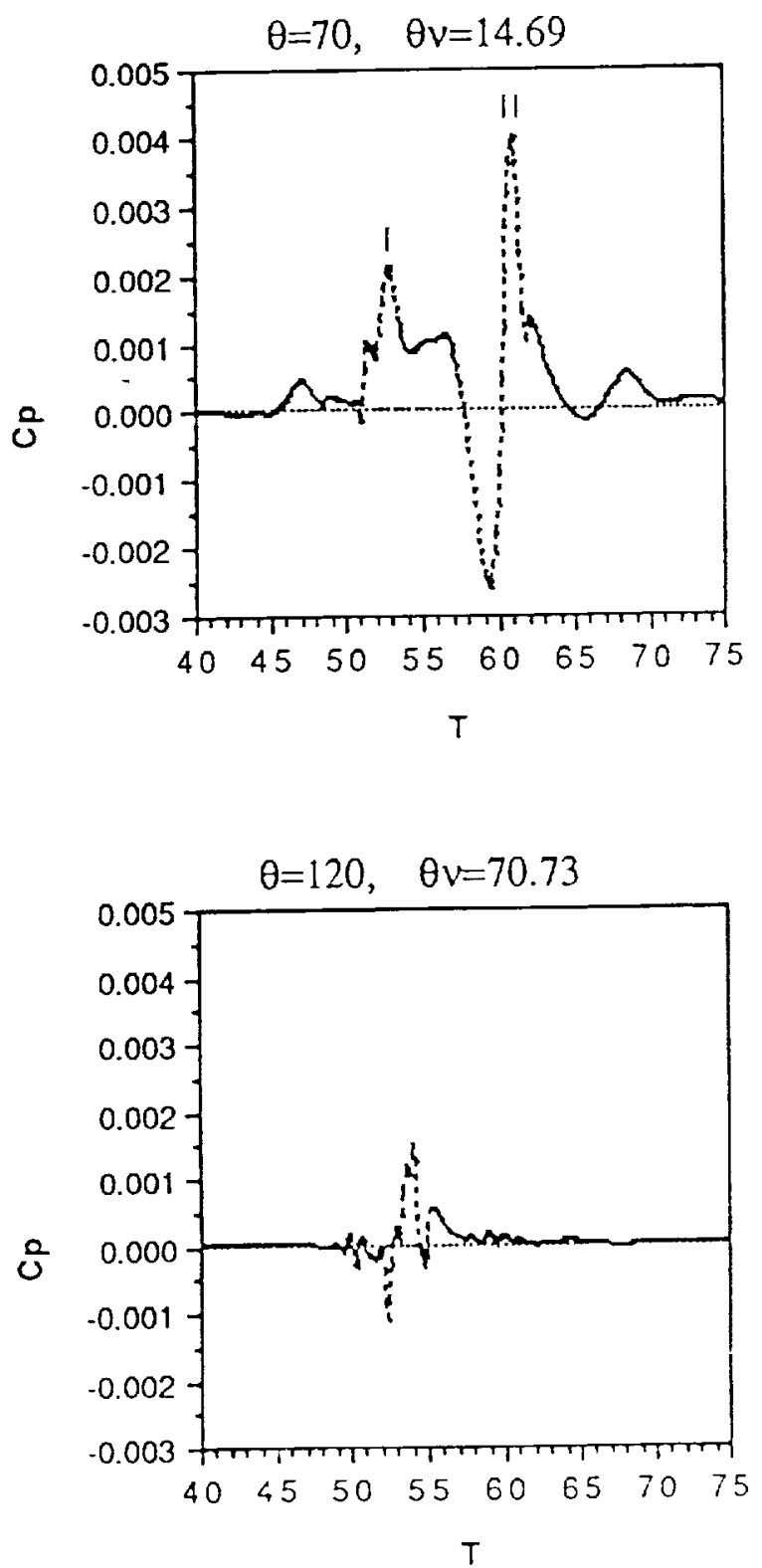

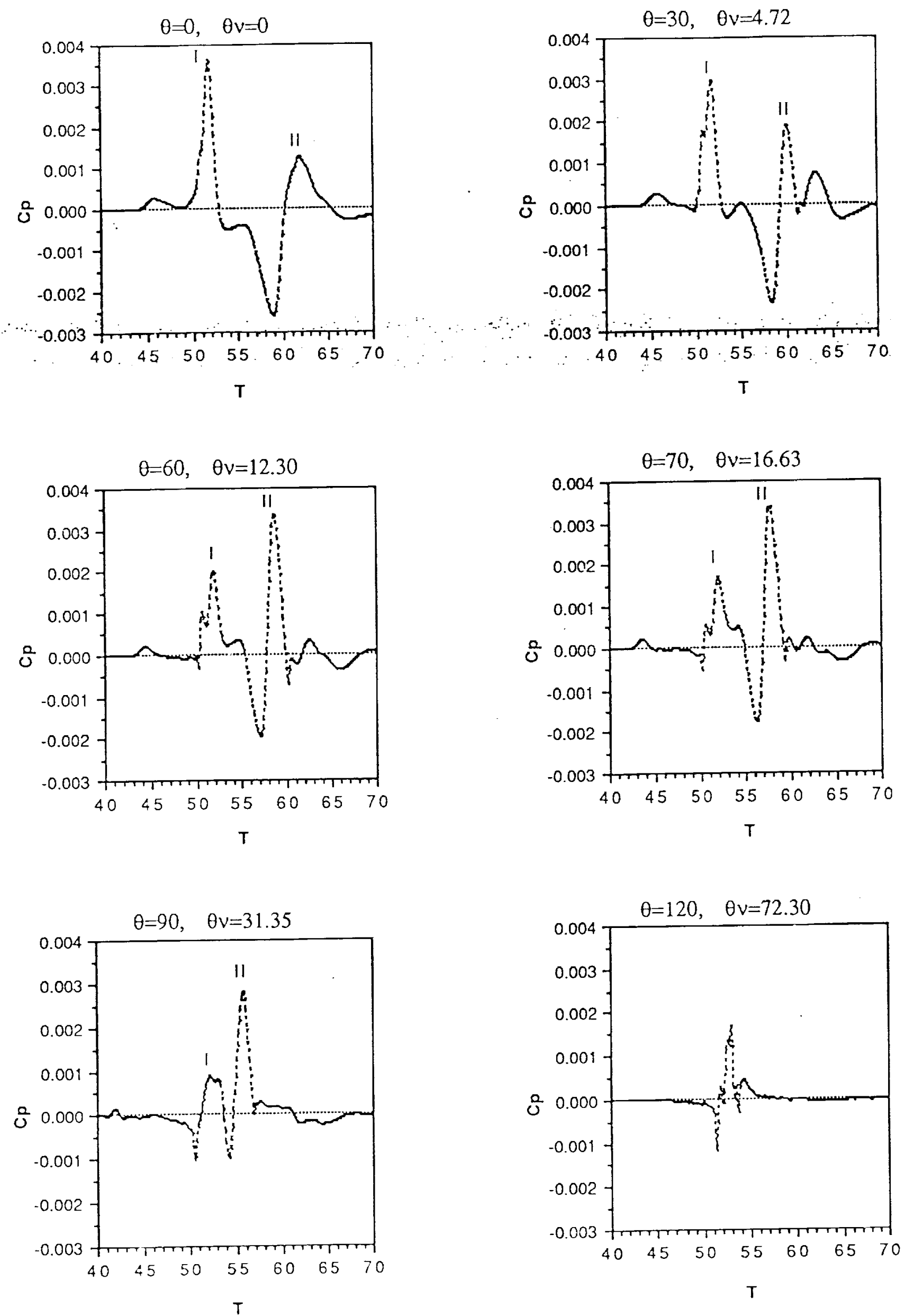

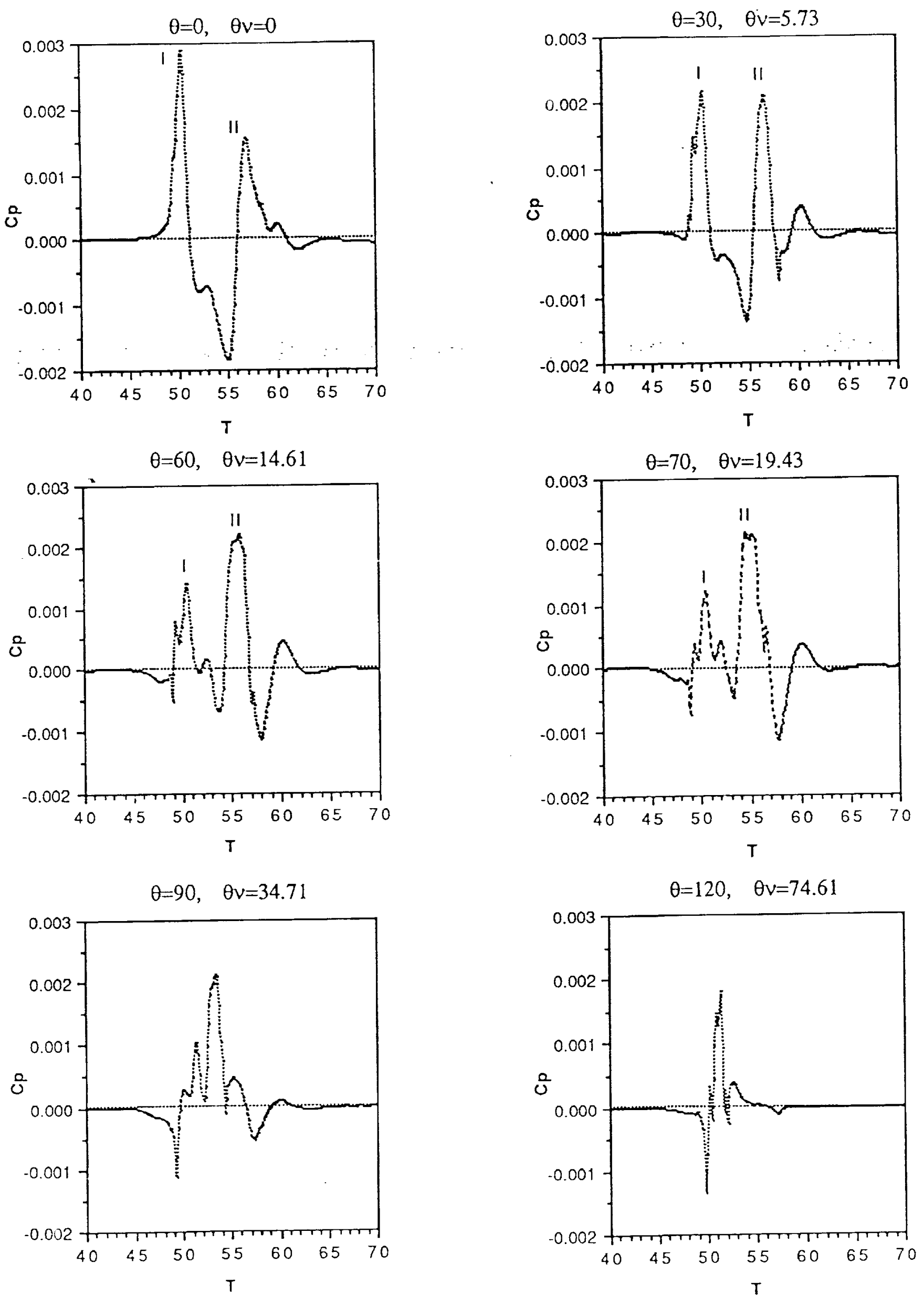

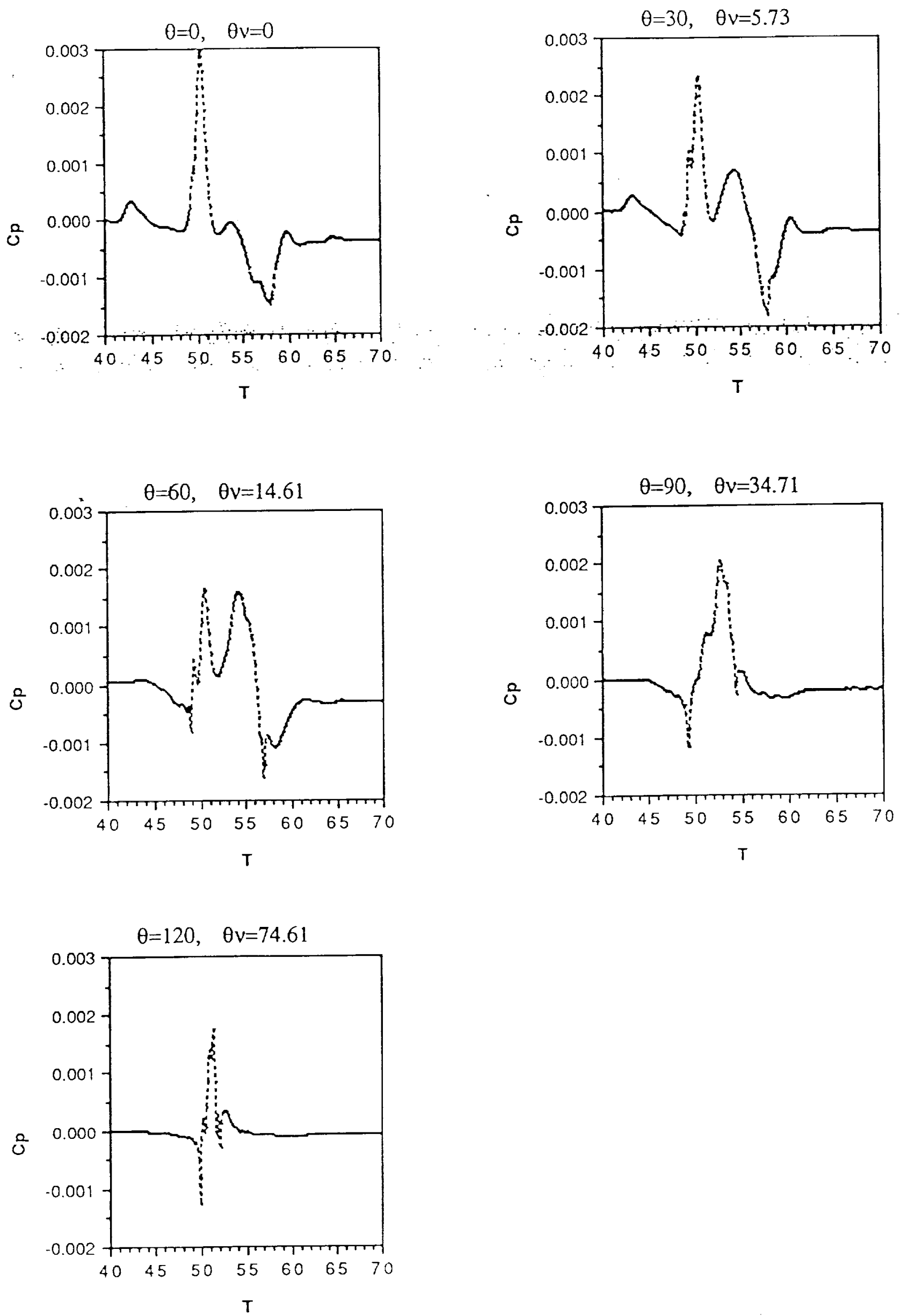


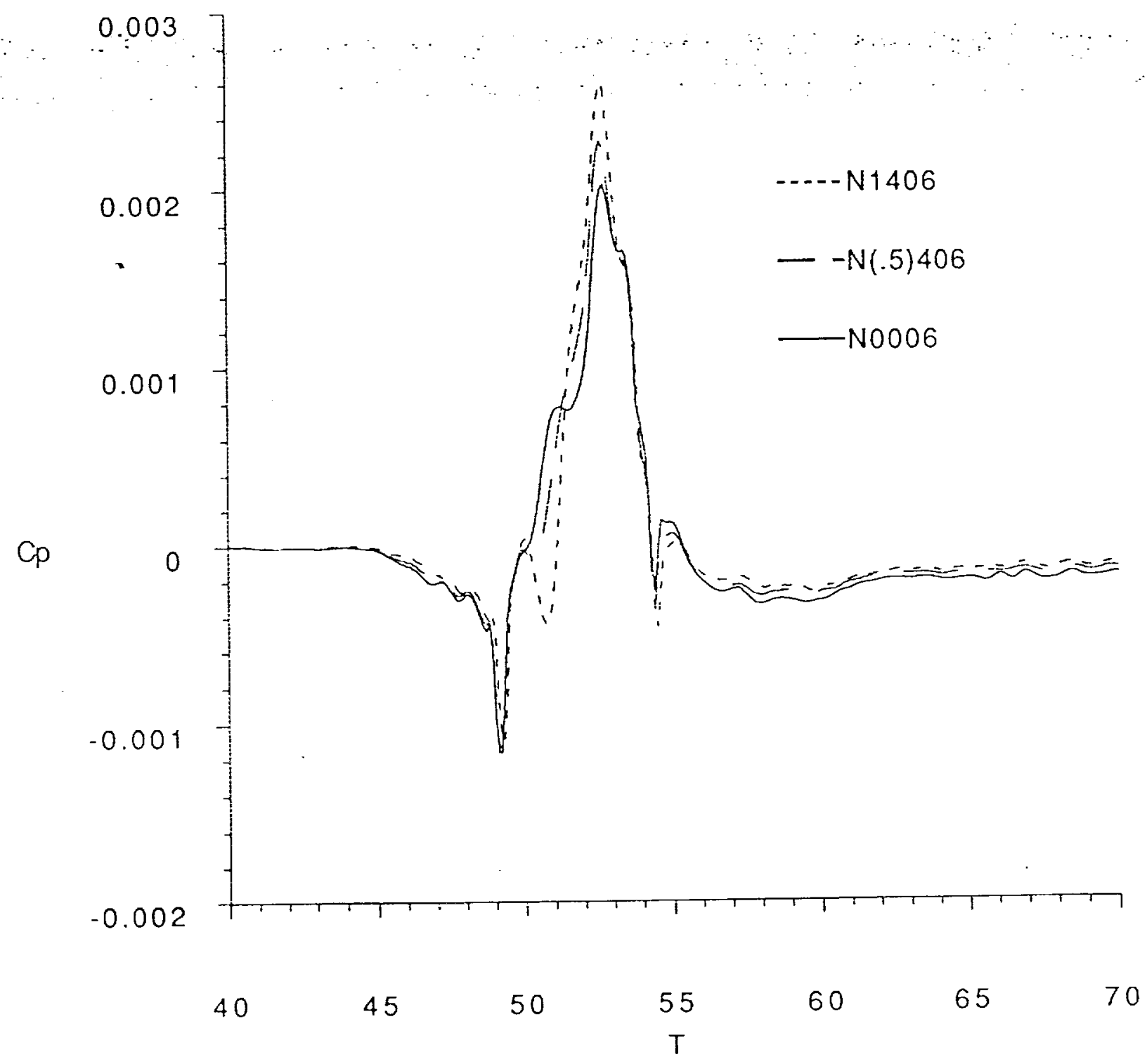

$\operatorname{tig} 19$ 


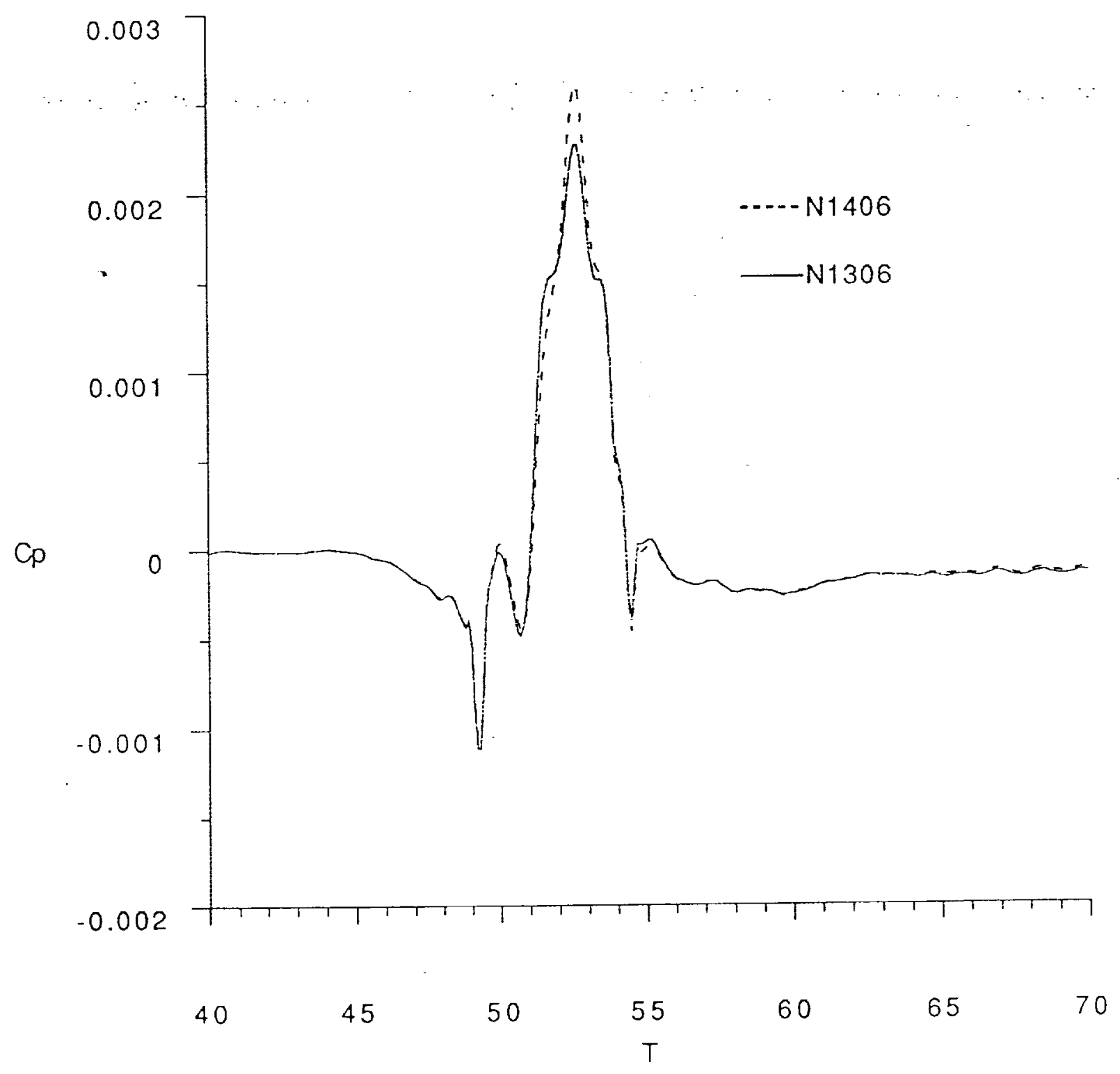

tig 20 